\title{
Diagnostic et aide dans un environnement d'apprentissage ouvert : Un exemple en chimie, SCHNAPS
}

\author{
François-Marie Blondel - Monique Schwob - Martial Tarizzo
}

Institut National de Recherche Pédagogique

Département Technologies Nouvelles

91, rue Gabriel Péri 92120 Montrouge

\begin{abstract}
RESUME. Cet article présente la conception et la réalisation d'un environnement ouvert pour l'apprentissage de la résolution de problèmes en chimie. Trois questions principales y sont étudiées : les outils mis à la disposition de l'élève, l'analyse de son activité et les aides que le système est en mesure de lui apporter. La conception de ces trois composantes de l'environnement s'appuie sur des analyses préalables des difficultés rencontrées, des formes de résolution des élèves et des demandes d'aide qu'ils ont formulées. Les outils de résolution calqués sur un modèle de calculette chimique permettent une aide indirecte et une observation détaillée de l'activité de l'élève. Le diagnostic, inspiré de la classification heuristique, cherche à reconnaître des formules et leurs conditions d'application dans les opérations et les relations exprimées par l'élève. Les informations qu'il fournit sur la résolution et indirectement sur les connaissances de l'élève permettent de proposer des interventions appropriées. L'organisation des aides et des interventions en assistants chargés d'un seul type d'aide offre une solution originale permettant de tenir compte des multiples facettes de l'assistance qu'un tel système est en mesure de prodiguer à un utilisateur.

ABSTRACT. This paper describes an open interactive learning environment for problem solving in chemistry. Three main points are discussed: the tools made available to the student, the analysis of his/her activity, and the help the system is capable to supply. The design of these three components of the environment relies upon a deep analysis of the difficulties the students encountered, the solutions they draw and the need for help they expressed. The solving tools that are based on a model of a chemistry calculator allow both an indirect help and a precise observation of the student's activity. The aim of the diagnosis which is derived from heuristic classification is to recognize formulas in the operations and relations written by the student. The results of the diagnosis which contains information on the student solving process are sufficient to build adequate help and advice. These are structured in independent entities named assistants which are in charge of only one kind of help.

MOTS-CLES : environnement ouvert, diagnostic, aide, conseil, résolution de problèmes, chimie KEYWORDS : open environment, diagnosis, help, advice, problem solving, chemistry
\end{abstract}




\section{Introduction}

Améliorer les capacités des élèves à résoudre les problèmes est souvent considéré comme l'un des objectifs majeurs de l'enseignement scientifique [REI 83] [JOS 93].

S'appuyant le plus souvent sur une modélisation de la résolution, de nombreux environnements de résolution de problèmes ont été développés en sciences [GRA 92] [BRU 97], mettant l'accent sur la représentation de la situation comme en mécanique [DIM 95], sur la recherche de la solution comme en algèbre dans APLUSIX [NIC 94], sur la construction de la solution comme en géométrie dans MENTONIEZH [PY 96], ou sur les méthodes de résolution comme en analyse dans ELISE [DEL 94].

Que la conception de ces environnements adopte un point de vue plus formel (celui des tuteurs) ou plus constructiviste (celui des micro-mondes), le caractère primordial de l'interaction a été maintes fois affirmé [DEL 94] [BER 95] [DUM 95] et régulièrement confirmé lors des expérimentations de systèmes opérationnels. La représentation du problème et de la situation, les outils mis à disposition de l'utilisateur pour manipuler cette représentation, sont au centre de la conception des environnements interactifs. Si ces questions ont été déjà largement abordées en mathématiques (voir [GRA 96] par exemple pour la géométrie), les travaux disponibles en physique et en chimie sont encore peu nombreux.

$\mathrm{Si}$, comme l'évolution des modes d'enseignement le laisse pressentir, on envisage un emploi de ces outils dans un contexte individuel d'autoformation, des aides directes et explicites sont nécessaires pour faciliter l'apprentissage. Dans un environnement ouvert, les systèmes d'aide, de guidage et de conseil ne peuvent être élaborés en fonction du contexte que si l'on dispose d'une analyse détaillée de l'activité de l'étudiant. Les difficultés rencontrées pour construire et maintenir un modèle de l'élève [SEL 91] [MCC 92] incitent à concevoir un diagnostic qui soit plus intégré aux outils de l'interaction [DIL 94] et qui fournisse tous les éléments nécessaires pour construire une aide ou une intervention appropriées.

Les formes que peut prendre une telle aide sont extrêmement variées : fonctionnement des outils, hypertexte conceptuel, conseils méthodologiques, explication de plans, dialogue,... Le choix d'un formalisme unique pour représenter ces aides contraindrait à n'en privilégier qu'une partie. Une situation d'apprentissage autonome impose de chercher à modéliser plusieurs aspects de l'assistance à l'utilisateur. Une analyse des demandes et des difficultés des utilisateurs apparaît alors comme absolument nécessaire.

Nous nous sommes proposés d'étudier ces questions d'interaction, de diagnostic et d'aide dans le cadre d'un projet d'environnement d'apprentissage en chimie [BLO 96], domaine qui combine à la fois des connaissances descriptives et des connaissances opérationnelles.

Dans une première partie, nous présentons les caractéristiques de ces problèmes et les difficultés de l'enseignement de cet aspect de la chimie et nous exposons la problématique générale du projet SCHNAPS. La deuxième partie contient la 
description des outils mis à disposition de l'élève ; la troisième présente les connaissances de référence et la résolution effectuée par le système. Dans la quatrième sont exposés les principes et la réalisation du diagnostic de l'activité de l'élève dans cet environnement. Les aides et les interventions du système sont détaillées dans la cinquième partie. Enfin, nous terminons par une présentation rapide des différentes versions de ce système et des expérimentations auxquelles elles ont donné lieu.

\section{Problèmes et problématique}

\subsection{Les problèmes de chimie quantitative}

En accord avec les programmes actuels de chimie de l'enseignement secondaire, nombre d'enseignants affirment la primauté d'une approche expérimentale, voire qualitative, de la chimie. Toutefois, les problèmes de chimie quantitative, ou problèmes stoechiométriques, sont un élément central de la coutume de l'enseignement. Il s'agit d'exercices construits à partir d'une réaction chimique et qui ont pour objet de mettre en œuvre les relations entre les différentes grandeurs attachées aux réactifs et aux produits de cette réaction.

Ces problèmes s'appuient sur une réaction chimique, le plus souvent unique et considérée comme totale. Les données de l'exercice, qui correspondent à une instanciation particulière de cette réaction, sont souvent des valeurs initiales ou finales de grandeurs extensives ${ }^{1}$ : masse, volume, quantité de matière d'un ou plusieurs réactifs ou produits. La question posée porte le plus souvent sur une grandeur liée soit à une quantité nécessaire d'un réactif soit à une quantité de produit formé.

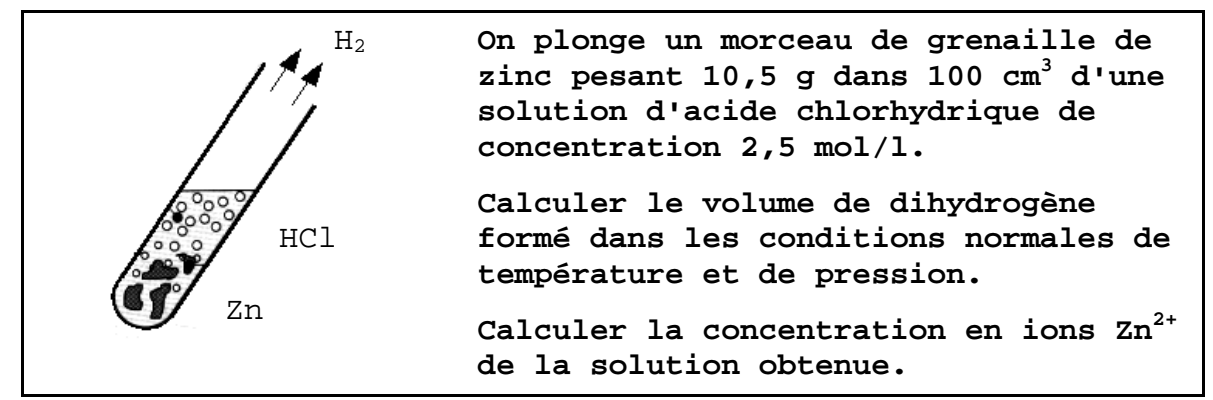

Figure 1. Exemple d'énoncé

Les énoncés, tels qu'ils apparaissent dans les manuels, impliquent un grand nombre de connaissances implicites liées à la représentation du problème et à sa résolution : nature des corps mis en jeu dans la réaction, équation-bilan, conditions stoechiométriques, conditions et caractéristiques physiques.

\footnotetext{
${ }^{1}$ On distingue les grandeurs extensives, comme la masse, qui dépendent de la quantité de substance, des grandeurs intensives, comme la masse molaire, qui se rapportent aux propriétés de la substance.
} 
La résolution implique l'écriture d'une équation-bilan équilibrée dans laquelle les coefficients ou nombres stoechiométriques indiquent les proportions de quantité de matière qui réagissent ou qui sont produites.

$$
2 \mathrm{H}_{3} \mathrm{O}^{+}+2 \mathrm{Cl}^{-}+\mathrm{Zn} \rightarrow \mathrm{H}_{2}+\mathrm{Zn}^{2+}+2 \mathrm{Cl}^{-}+2 \mathrm{H}_{2} \mathrm{O}
$$

Les méthodes proposées par les enseignants et les manuels sont assez semblables dans leur principe, même si elles varient sensiblement dans leur présentation. Elles utilisent toujours la quantité de matière, grandeur considérée comme centrale dans toutes ces résolutions. Elles s'appuient toutes sur l'expression d'une proportionnalité directement déduite de l'équation-bilan, comme dans l'exemple suivant :

Quantité $\mathrm{H}_{2}$ finale = Quantité $\mathrm{H}_{3} \mathrm{O}^{+}$initiale / 2

On ne rencontre qu'exceptionnellement l'utilisation de relations traduisant directement des lois de conservation de la masse, conservation que les élèves mobilisent toutefois, à bon escient ou non, même lorsqu'on ne les leur a pas enseignées.

\subsection{Les difficultés connues}

De nombreux travaux de didactique témoignent de la permanence des questions soulevées par ces problèmes dans l'enseignement dans plusieurs pays. Ces questions restent d'actualité jusqu'à un niveau d'enseignement élevé comme le rappelle une analyse récente sur la maîtrise de l'équation-bilan par les étudiants en DEUG [MEY 94].

Certains auteurs dont [KRA 82], ont analysé de façon détaillée les difficultés des élèves au cours de la résolution des problèmes de stoechiométrie. Ils en tirent la conclusion que les enseignants, trop experts en la matière, omettent très souvent les étapes d'analyse et de décomposition pour enseigner une approche systématique de la résolution, éludant ainsi les points délicats de celle-ci.

Les difficultés des élèves et des étudiants qui peuvent en partie être prises en compte dans un environnement informatisé relèvent principalement de :

- la diversité et la multiplicité des connaissances indirectement mises en jeu

Les énoncés des problèmes font appel à un très grand nombre de composés et de réactions chimiques. Un environnement doté de ce type de connaissances peut contribuer à réduire la charge cognitive induite par cette diversité.

- la complexité du problème pour un élève débutant

Cette complexité est liée au nombre de relations, de grandeurs et d'objets manipulables au cours de la résolution; elle disparaît avec la maîtrise d'une méthode de résolution, mais réapparaît immédiatement dès que les problèmes posés sortent des situations canoniques. Des outils de représentation du problème et de manipulation de cette représentation peuvent faciliter la prise en charge de cette complexité.

- la construction des concepts de quantité de matière et de réaction chimique La construction et le fonctionnement du concept de réaction chimique dépendent de la construction du concept scientifique de substance qui ne semble pas complètement acquis à la fin de l'enseignement secondaire [STA 90]. Quant au 
concept de quantité de matière, il est reconnu comme difficile, mais a été très peu étudié. Des connaissances et des explications sur ces concepts sont probablement indispensables dans un environnement d'apprentissage dans ce domaine.

\subsection{Les phases principales de la résolution}

Afin de préciser quelles fonctions on peut attribuer à un environnement interactif d'aide à la résolution et pour faciliter la comparaison avec d'autres domaines ([TRI 96] en géométrie), nous distinguerons trois phases principales dans l'activité de résolution de ce type de problèmes : une phase d'analyse, une phase d'exploration, et une phase de rédaction. Cette distinction ne suppose pas que ces trois phases soient obligatoirement séparées dans le temps.

La phase d'analyse correspond au passage de l'énoncé à une représentation explicite, souvent plus formelle du problème ou de la situation décrite dans l'énoncé. En sciences physiques, cette analyse a été étudiée en détail par G. Tisseau [TIS 90] qui en a proposé une modélisation dans le cas de la thermodynamique. L'extension de cette modélisation à un autre domaine demanderait un travail important d'expression linguistique des concepts sans que pour autant l'usage d'un tel modèle pour l'apprentissage n'ait été ni proposé ni validé. Les questions qui se posent au cours de cette phase concernent la représentation graphique, le langage de description (il n'y a pas toujours de langage précis disponible, comme en mathématiques), et enfin le contrôle de la représentation ou de la description obtenue, comme cela se pose pour l'acquisition et la construction de la figure en géométrie [DES 96]. Nous ne les avons que partiellement abordées au cours de notre projet.

La phase d'exploration au cours de laquelle l'élève recherche les caractéristiques qui vont contribuer à la résolution, est celle qui a été la plus largement étudiée dans les EIAO. En chimie, D. Cabrol et ses collaborateurs du LARTIC ont proposé plusieurs environnements d'exploration ${ }^{2}$ construits autour d'une expertise, d'une simulation ou d'une base de données et incluant des systèmes de conseils [CAB 88] [CAB 91] [CAB 94]. Notre travail s'inspire directement de ces environnements qui ont connu un réel succès auprès des enseignants et des étudiants.

La phase de rédaction qui a pour but de produire une trace lisible de la résolution, est celle qui est la plus proche de l'activité scolaire habituelle. Quelques exemples de systèmes réactifs, comme le tuteur MENTONIEZH qui porte sur l'exposé de la démonstration en géométrie [PY 96], analysent la production de l'élève, élaborent un diagnostic et prodiguent évaluation et conseils. Notre intention était de concevoir un tel système dans une perspective d'aide à la résolution.

\subsection{Le projet Schnaps}

Connaissant les difficultés de l'enseignement de cet aspect de la chimie, l'objectif fixé initialement à ce projet était double :

\footnotetext{
${ }^{2}$ qualifiés par leurs auteurs de partenaires de résolution de problèmes.
} 
- Étudier les mécanismes de résolution, les difficultés des élèves et les explications proposées par les enseignants.

- Proposer des outils d'aide à l'apprentissage, principalement axés sur l'exploration de la situation et la rédaction de la solution,

Nous avons fait le choix d'un environnement interactif ouvert dans lequel l'élève définit lui-même le problème posé, et s'exerce à résoudre tout en bénéficiant d'un guidage discret ${ }^{3}$. Cet environnement cherche à faciliter le travail de l'élève par la fourniture d'outils et d'aides appropriés, et celui de l'enseignant par une analyse du travail de l'élève et une prise en charge partielle de la fonction de conseil. Dans ce type d'environnement, l'élève et le système ont accès aux mêmes connaissances de référence pour explorer la situation et construire une solution.

La méthode employée dans ce projet a consister à alterner des phases de conception et des phases d'expérimentation. L'analyse des activités d'élèves et des pratiques d'enseignants a été utilisée dans les phases de conception (interface utilisateur, diagnostic des actions, aides et interventions) ; l'expérimentation de versions intermédiaires a fourni des corpus d'interactions utilisés par la suite pour concevoir le diagnostic et les aides, et a permis de contrôler l'adéquation des outils proposés aux objectifs fixés et au public concerné (élèves du secondaire principalement). Trois étapes principales ont marqué le déroulement du projet : la conception d'un environnement minimal, son expérimentation et la conception du diagnostic, et enfin, la conception du système d'aide.

Nous présentons dans la suite de cet article l'état actuel du projet.

La dernière version de l'environnement qui prend en compte les principales évolutions du projet depuis son origine comprend :

- une interface de définition du problème,

- une interface de recherche et de rédaction de la solution,

- une base de données sur les corps et les réactions chimiques,

- un résolveur à base de formules et de plans,

- un module de diagnostic des actions de l'élève,

- des fonctions d'aide et de conseil.

\footnotetext{
${ }^{3}$ Le guidage discret (coaching) est employé pour désigner les logiciels d'apprentissage ouverts qui prennent l'initiative d'apporter une aide ou un conseil à l'utilisateur au cours de son travail.
} 


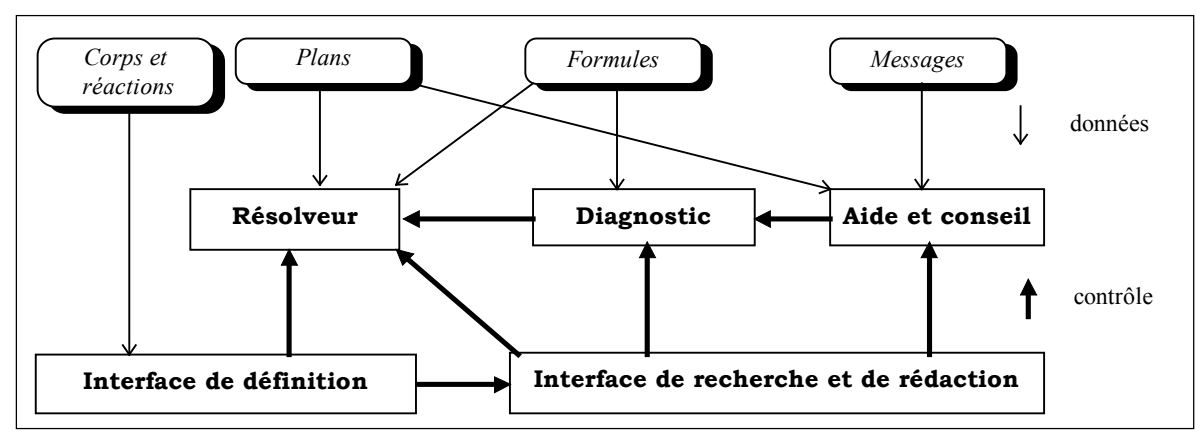

Figure 2. Architecture générale de l'environnement

\section{Outils pour l'élève}

\subsection{Leur rôle dans l'interaction}

Les outils proposés pour définir le problème et le résoudre ont pour rôle de :

- faciliter le travail de l'élève, en explicitant les étapes et en mettant en avant les représentations,

- renseigner les concepteurs et les didacticiens, en décomposant l'activité de recherche et de résolution.

Par rapport au déroulement de la résolution décrit plus haut, l'interaction est décomposée en deux phases, impliquant par conséquent deux ensembles d'outils. Durant la première phase, dite de définition, l'élève décrit la situation c'est-à-dire la réaction et ses conditions. Ensuite, cette situation étant correctement décrite, au cours de la deuxième phase, dite de recherche et de rédaction, l'élève explore les valeurs des grandeurs en jeu et explicite les relations successives entre grandeurs qui mènent à la solution.

L'interaction durant la première phase a été conçue avec un souci d'efficacité afin d'aboutir assez facilement à la résolution avec une description acceptable; en conséquence, la conduite de l'interaction y est assez directive. Elle garantit que l'élève ne commence pas une recherche de solution sur la base d'une réaction impossible ou incomplètement définie. En revanche, la recherche et la rédaction de la solution ont été conçues de manière intégrée et sous une forme très ouverte et non directive, ceci pour recueillir des informations pertinentes sur les comportements et les limites des élèves devant de tels outils et sur les capacités du système à analyser une rédaction assez libre.

La conception des outils de recherche et de rédaction à été effectuée après une analyse de traces écrites et d'enregistrements de résolution à voix haute, corpus qui a servi également par la suite pour la conception du diagnostic. 


\subsection{Les outils de définition}

Le but de cette phase de définition est de passer du texte de l'énoncé que l'élève est seul à connaître, à une représentation complète du problème, c'est-à-dire de la réaction chimique, de ses constituants, et des conditions dans lesquelles elle se déroule.

Durant cette phase, l'utilisateur effectue trois actions principales :

- la définition des corps et de la réaction

L'élève définit un corps en indiquant son nom ou sa formule chimique et l'introduit dans la réaction comme réactif ou comme produit.

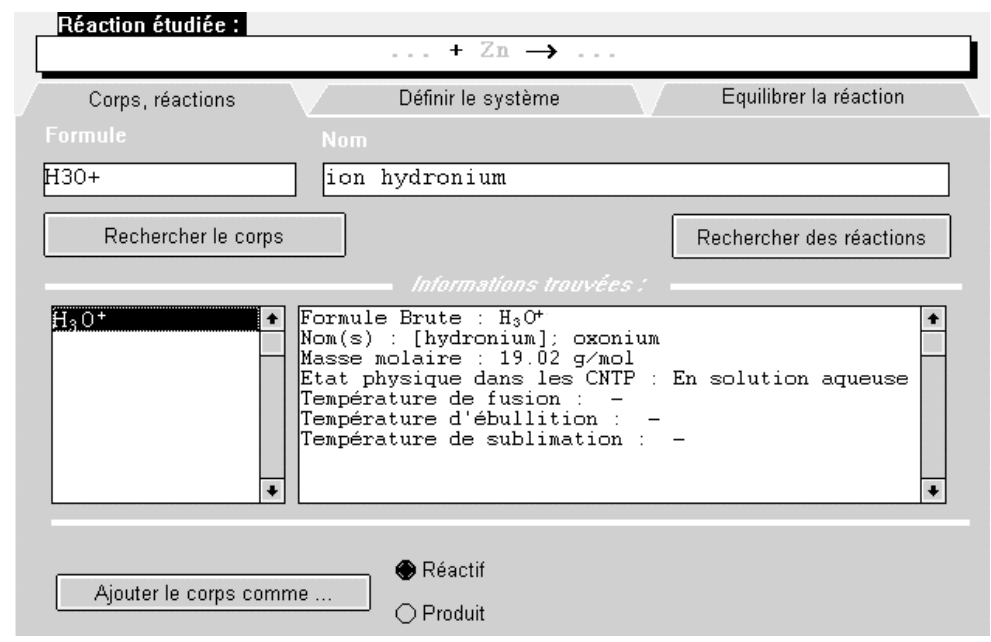

Figure 3. Introduction d'un corps

- la définition du système physico-chimique

La définition du système consiste à indiquer dans quelle phase (solide, liquide, gaz, ou solution) se trouve chacun des composés avant la réaction et après la réaction.

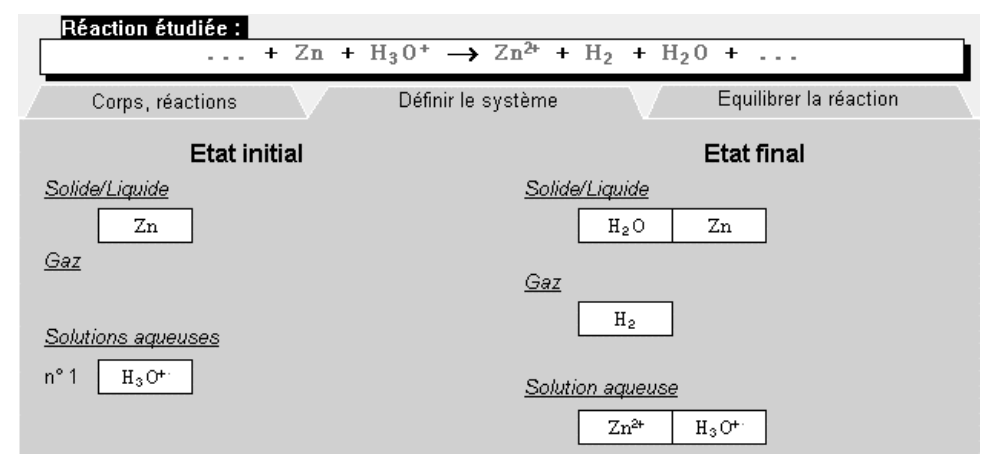

Figure 4. Les deux états du système une fois défini 
- l'équilibrage de l'équation-bilan

L'équilibrage consiste à indiquer les coefficients ou nombres stoechiométriques de l'équation-bilan, c'est-à-dire les proportions de quantités de matière des corps qui réagissent. Le système vérifie la cohérence des coefficients introduits par l'élève et lui propose une solution le cas échéant. Ce calcul ou cette vérification sont effectués par la propagation de contraintes exprimant les relations en jeu.

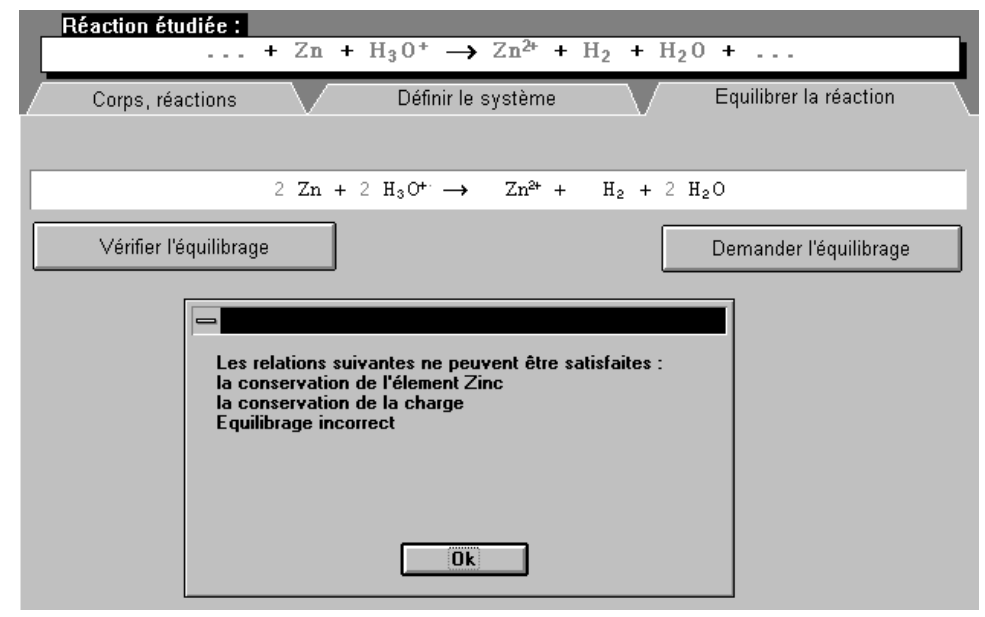

Figure 5. Équilibrage par l'élève et vérification de la cohérence

A l'issue de cette phase, un contrôle est nécessaire pour deux raisons.

D'une part, il existe un certain nombre de conditions à respecter pour que le problème posé en termes numériques soit soluble, c'est-à-dire pour que l'on puisse implanter un ensemble de relations qui ne soit pas contradictoire; ces conditions qui expriment des principes de conservation sont testées avant l'équilibrage et au cours de cet équilibrage. Ils constituent en quelque sorte les invariants de la réaction chimique.

D'autre part, il apparaît comme totalement inconcevable (en particulier pour les enseignants) de travailler sur une réaction qui n'existerait pas. Ce dernier contrôle s'effectue de manière indirecte par des recherches dans la base de corps et de réactions. Toute contradiction avec les informations de la base est signalée à l'élève, de même que l'absence d'informations correspondantes dans la base.

\subsection{Les outils de recherche et de rédaction}

Les outils proposés pour cette activité de recherche et de rédaction de la solution s'apparentent à une feuille de brouillon couplée à une calculette chimique.

Cette métaphore a pour objectif d'assurer une homogénéité et une cohérence plus grandes entre les différentes actions de l'élève. Assez peu utilisée pour des environnements en physique, elle a été employée en particulier par D. Bierman [BIE 92] pour analyser le travail de l'élève car elle fournit les moyens de rendre explicites des intermédiaires qui sont habituellement implicites pour l'élève. 
L'interface comprend une fenêtre de rédaction dans laquelle l'élève peut noter, définir, relier, calculer, commenter, etc. à l'aide de fonctions. Ces fonctions réalisent une décomposition de la tâche de recherche et de rédaction en tâches plus élémentaires. Chaque appel à une fonction offre un cadre de dialogue dans lequel l'élève introduit les éléments particuliers à sa proposition. La validation entraîne l'insertion de la ligne correspondante dans la feuille de rédaction.

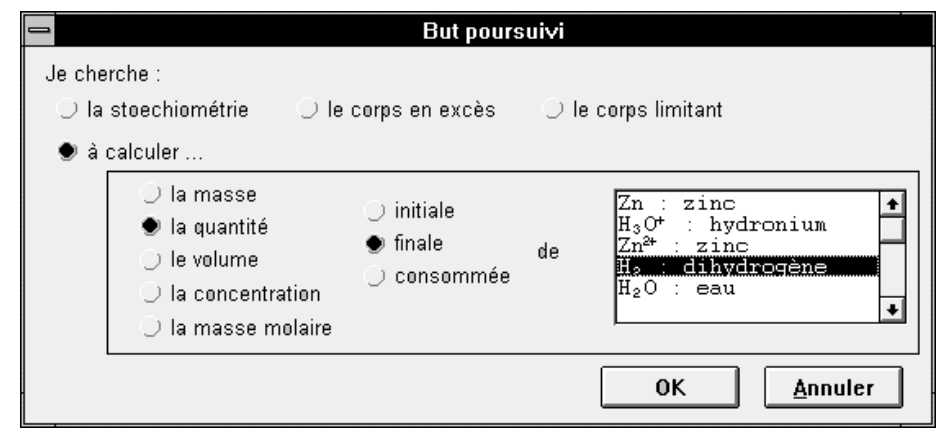

Figure 6. Cadre de dialogue pour indiquer un but

L'utilisateur peut éditer les différents éléments de cette rédaction par insertion, suppression ou réévaluation. La réévaluation consiste à reproduire l'appel d'une fonction dans le nouveau contexte tenant compte des insertions et des suppressions effectués depuis l'évaluation précédente.

L'outil de résolution intègre deux composantes, l'une relevant de l'édition de textes structurés qui a pour objet de déclarer, et l'autre du résolveur d'équations.

\begin{tabular}{|l|l|}
\hline Enoncé & $\begin{array}{l}\text { pour déclarer les valeurs numériques des grandeurs fixées } \\
\text { dans l'énoncé, le plus souvent une ou plusieurs grandeurs } \\
\text { de réactifs ou de produits. }\end{array}$ \\
\hline Question & $\begin{array}{l}\text { pour indiquer la question posée dans l'énoncé, le plus } \\
\text { souvent la valeur d'une grandeur extensive d'un autre réactif } \\
\text { ou produit. }\end{array}$ \\
\hline But & $\begin{array}{l}\text { pour indiquer ce que l'on cherche à faire dans les actions } \\
\text { suivantes }\end{array}$ \\
\hline Stoechiométrie & pour déclarer les conditions stoechiométriques \\
\hline Commentaire & pour insérer un texte libre dans la rédaction \\
\hline
\end{tabular}

Figure 7. Outils de déclaration

Comme pour une résolution écrite, la même action peut quelquefois être effectuée de plusieurs manières différentes, soit en combinant les outils, soit en choisissant un outil plutôt qu'un autre; il existe un recouvrement partiel entre certains outils (relation et calcul par exemple). 


\begin{tabular}{|l|l|}
\hline Définition & pour nommer une grandeur du problème \\
\hline Relation & $\begin{array}{l}\text { pour introduire sous une forme canonique une relation } \\
\text { algébrique entre grandeurs, désignées par leur nom ou par } \\
\text { leur valeur }\end{array}$ \\
\hline Calcul & $\begin{array}{l}\text { pour effectuer un calcul sur des valeurs et des grandeurs } \\
\text { connues et éventuellement en affecter le résultat à une autre } \\
\text { grandeur. }\end{array}$ \\
\hline Recalcul & $\begin{array}{l}\text { pour évaluer à nouveau un calcul, voire une déclaration, en } \\
\text { cas de changement dans les valeurs numériques des } \\
\text { grandeurs définies }\end{array}$ \\
\hline
\end{tabular}

Figure 8. Outils de transformation

Les grandeurs nommées avec l'outil de définition peuvent être utilisées dans les relations ou les expressions à calculer. Les expressions à calculer sont évaluées en tenant compte des valeurs des grandeurs nommées, ce calcul s'effectuant en tenant compte des unités associées, opérant au besoin les conversions nécessaires. Cet ensemble de fonctionnalités relèvent de la "calculette chimique".

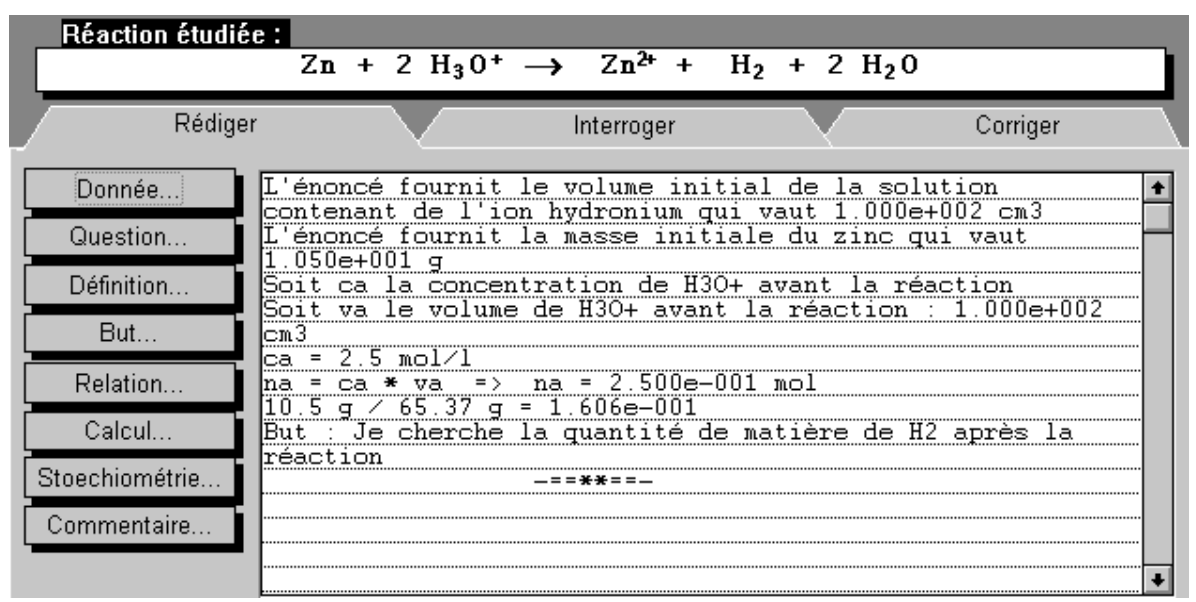

Figure 9. Feuille de rédaction en cours de travail

\section{Les connaissances de références et le résolveur}

\subsection{Connaissances de référence}

La représentation $\mathrm{du}$ problème s'articule autour de quatre concepts fondamentaux : les corps chimiques, les grandeurs, leurs relations et le système chimique.

L'ensemble des connaissances nécessaires au contrôle de la définition du problème sont rassemblées dans une base de données. Ces connaissances concernent 
les corps chimiques (formule et état physique) et les réactions chimiques (réactifs et produits).

Certaines de ces connaissances ont une portée générale ("tous les alcanes réagissent avec l'oxygène pour produire du gaz carbonique et de l'eau") et pourraient être représentées sous forme de règles. Cependant cette représentation n'est actuellement possible et disponible que dans des secteurs très spécialisés de la chimie. D'autres sont strictement factuelles, comme la température d'ébullition du butane sous pression normale, ce qui permet d'en déduire son état physique. La solution adoptée consiste à constituer une base de données à partir des ouvrages de référence disponibles.

La constitution de la base des corps a été effectuée à partir d'une analyse des manuels scolaires pour déterminer une première liste de corps. Les informations associées à chaque corps ont été limitées à ce qui est nécessaire pour l'interaction avec l'élève (formule semi-développée, noms) pour la définition de la réaction chimique et pour la résolution (type, formule brute, charge, masse molaire, état physique).

La constitution de la base de réactions a aussi été effectuée à partir d'une analyse des mêmes manuels; mais le nombre des réactions présentes dans les manuels n'est pas suffisant pour offrir une couverture minimale du domaine. Elle a été complétée de façon un peu plus systématique pour certaines réactions de la chimie organique. Cependant si les réactions les plus couramment utilisées y sont présentes (400 réactions environ), la base obtenue ne permet pas encore de couvrir la plupart des réactions qu'un enseignant peut proposer à un élève.

\subsubsection{Corps}

A chaque corps sont associées des propriétés (formule, nom, état physique, charge, ...), des grandeurs (masse, volume, masse-molaire, concentration, ...) et des relations.

Exemple : Propriétés de l'ion $\mathrm{H} 3 \mathrm{O}+$

$\begin{array}{ll}\text { Formule } & \mathrm{H} 30+ \\ \text { Nom } & \text { ion hydronium } \\ \text { Etat physique } & \text { en-solution } \\ \text { Charge } & +1\end{array}$

\subsubsection{Grandeurs}

Les grandeurs utilisées sont associées soit à un corps, soit à une phase. Dans un système physique général, les grandeurs évoluent dans le temps. Dans la représentation de la réaction complète, on ne s'intéresse a priori qu'à deux instants : Initial et Final. Pour exprimer plus facilement les relations introduites par la réaction chimique dans le cas le plus général, nous avons introduit un troisième instant, celui de la réaction lui-même, qui caractérise les valeurs qui réagissent effectivement. Cet instant est noté Reagit. Ainsi chaque grandeur est représentée par un triplet: <nom> <corps> <instant>. 
Exemples :

(Volume H2 Final)

(Masse Zn Initial)

(Quantite $\mathrm{Zn}$ Reagit)

\subsubsection{Relations et Formules}

Les relations expriment principalement les lois physiques et chimiques qui définissent des grandeurs ("la masse volumique d'un corps est le rapport de sa masse à son volume") ou qui lient les grandeurs du problème entre elles ("les quantités de matière qui interviennent dans la réaction sont proportionnelles aux coefficients de l'équation-bilan").

Elles expriment également des contraintes élémentaires qui découlent de la représentation physique du problème ("dans une réaction en solution, le volume final de la solution est égal à la somme des volumes initiaux des solutions contenant les réactifs").

La représentation des relations par des formules permet de les traduire quantitativement pour calculer les valeurs des grandeurs du problème mais surtout de les utiliser pour analyser les rédactions des élèves. Cette représentation est de la forme :

(= <expression gauche> <expression droite>)

où <expression gauche> et <expression droite> sont des expressions algébriques dont les opérandes sont les grandeurs ou les constantes du problème.

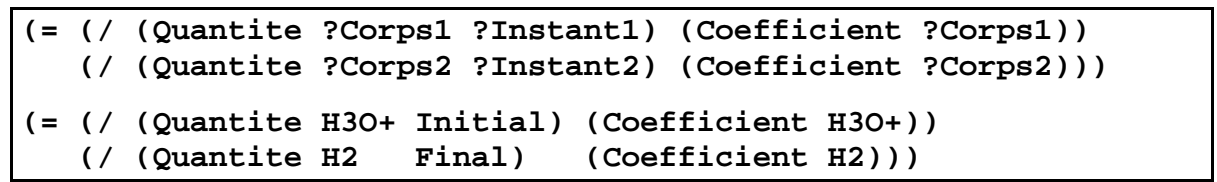

Figure 10. Exemples de relations, la première générale et la seconde instanciée pour deux des corps de la réaction

\subsubsection{Système physico-chimique}

Bien que les énoncés et les solutions-types des problèmes fassent une impasse à peu près totale sur ce point, il est nécessaire de définir dans quel système la réaction se déroule afin de pouvoir écrire les relations qui marquent les échanges d'un point de vue physique : combien y a-t-il de corps à l'état gazeux avant et après la réaction ? dans quelle enceinte ? quel en est le volume total ? combien y-a-t-il d'espèces en solution avant la réaction ? et après ? dans quel volume?

Afin de pouvoir traiter les problèmes dans leur plus grande généralité, nous avons adopté la notion de système chimique introduite initialement par D. Cabrol pour un résolveur de problèmes de chimie [CAB 87]. 


\begin{tabular}{|c|c|c|c|}
\hline & & état initial & état final \\
\hline \multirow[t]{4}{*}{ phases } & solide & $\mathrm{zn}$ & $\mathrm{Zn}$ \\
\hline & liquide & & H2O \\
\hline & gaz & & H2 \\
\hline & solution & $\mathrm{H} 3 \mathrm{O}+, \mathrm{Cl}-$ & $\begin{array}{l}\mathrm{Zn++}, \mathrm{H} 3 \mathrm{O}+, \\
\mathrm{Cl}-\end{array}$ \\
\hline \multirow[t]{2}{*}{ grandeurs } & température & $25^{\circ} \mathrm{C}$ & $25^{\circ} \mathrm{C}$ \\
\hline & pression & 1 bar & 1 bar \\
\hline
\end{tabular}

Figure 11. Système chimique correspondant à la réaction de l'acide chlorhydrique sur le zinc

Un système chimique comporte deux états : l'état initial et l'état final. Chaque état est caractérisé par la liste de ses phases constituantes et les conditions physiques de température et de pression. Chaque phase est caractérisée par sa nature (Solide, Liquide, Gaz, Solution) ainsi que par la liste de ses constituants (Corps).

\subsection{Résolveur}

La résolution des problèmes de chimie quantitative peut très bien s'effectuer au moyen d'un algorithme qui applique la méthode la plus générale pour résoudre ces problèmes. Une approche déclarative permet cependant une plus grande souplesse d'adaptation de la résolution aux différents problèmes et une plus grande facilité d'assistance à l'élève.

Le résolveur, construit sur des caractéristiques d'origine pédagogique, joue un double rôle ; il sert de référence pour analyser l'activité de l'élève (diagnostic), et il permet de donner une solution ou plutôt un résultat correspondant aux données qui ont été introduites (interrogation par l'élève), et accessoirement d'expliquer (car les élèves s'intéressent plus à leur propre résolution pour y déceler là où ils se sont "trompés", plutôt qu'à celle proposée par le résolveur).

Les qualités attendues du résolveur sont les suivantes:

- robustesse : fonctionner quel que soit le jeu de données introduit par l'élève,

- étendue : calculer tout ce qui est calculable en fonction des données connues,

- explicabilité : rendre compte d'un résultat dans un langage compréhensible.

La résolution dans SCHNAPS est effectuée par un système de propagation de contraintes. Ce système permet de calculer un grand nombre de valeurs de grandeurs avec une bonne efficacité.

Les contraintes correspondent à la traduction des relations entre grandeurs, relations instanciées par les grandeurs du problème. Elles sont implantées pour une partie au moment de la définition de la situation, et résultent pour l'autre partie de la mise en application d'une méthode de résolution, représentée par des plans. 


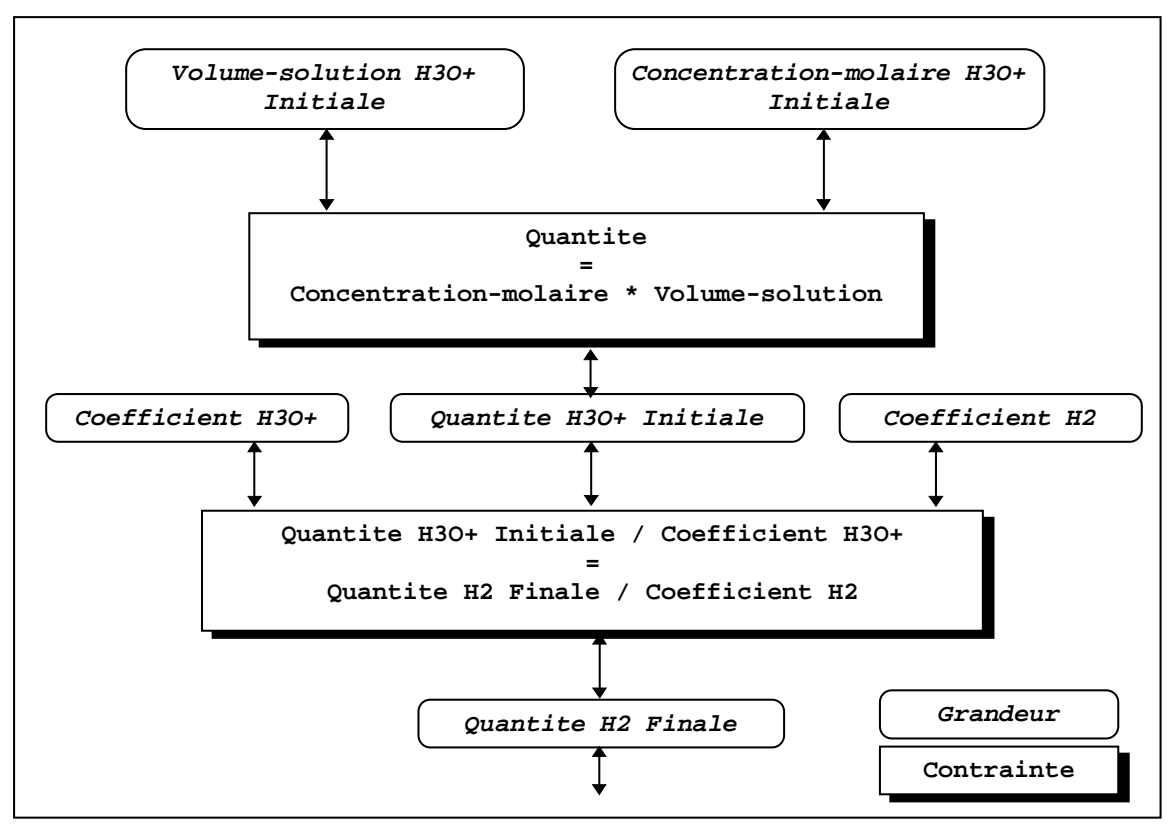

Figure 12. Graphe partiel représentant deux contraintes reliant six grandeurs

Les plans expriment une analyse des méthodes de résolution possibles, qui (1) vérifie que les données disponibles sont suffisantes et cohérentes, et (2) fixe la grandeur dont la valeur sera propagée et (3) implante les relations construites à partir de cette grandeur.

Ces plans représentent une décomposition hiérarchique des tâches, ou actions possibles. Il n'y a pas de recherche heuristique, la seule question qui se pose est de proposer le bon plan en fonction de ce que l'on souhaite montrer à l'élève, et bien sûr en fonction du problème. La hiérarchie de plans que cette représentation permet de générer à partir de l'objectif initial est à rapprocher du graphe des tâches que $\mathrm{F}$. Pachet propose pour décrire les actions significatives dans le système de conseil EPITALK [PAC 94].

\begin{tabular}{|ll|}
\hline nom & fixer-seul-reactif-avec-valeurs-initiales \\
objectif & rechercher le corps qui fixe d'après les données \\
conditions & il existe un seul réactif avec des valeurs initiales \\
il n'y a pas de corps avec valeur consommée ou produite & \\
actions & Faire choisir ce réactif pour fixer les quantités \\
& fixer sa quantité finale à 0
\end{tabular}

Figure 13. Un des plans de choix du corps qui fixe les quantités de la réaction 


\section{Diagnostic des activités de l'élève}

\subsection{Introduction}

Quand les actions de l'élève ne posent pas de problème d'interprétation, un diagnostic des connaissances conçu en vue d'une modélisation de l'élève est possible [NGU 93] [PY 95] [TAL 92] mais reste le plus souvent difficile voire dans certains cas impossible [SEL 90].

Dans un environnement ouvert qui offre à l'élève un large éventail de moyens d'expression de sa recherche et de sa résolution, le premier et principal problème est de déterminer avec suffisamment de précision ce que l'élève a fait, c'est-à-dire de trouver une première interprétation à des actions élémentaires elles-mêmes peu significatives. En ce sens, on peut considérer ce diagnostic comme plus proche d'un diagnostic du comportement nécessitant le cas échéant une analyse didactique pour identifier les observables [BAL 94]. Cette situation se retrouve par exemple dans les environnements de type micro-monde comme CABRI, ou dans ceux qui font appel à des simulations comme HIPOCAMPE qui traite des équilibres thermodynamiques [FOR 93].

Les idées principales que nous retenons pour le diagnostic dans un environnement interactif ouvert, sont les suivantes :

- la finalité du diagnostic est de fournir toutes les informations nécessaires pour une intervention adaptée à la situation, et accessoirement de proposer une analyse détaillée de la résolution de l'élève, en vue d'une évaluation,

- le diagnostic porte principalement sur le comportement et indirectement sur les connaissances, étant donné que la complexité des actions possibles ne peut être uniquement expliquée par l'application de procédures,

- des analyses didactiques préalables sur les résolutions des élèves sont nécessaires pour décrire les éléments pertinents des comportements et proposer une catégorisation qui permette de les relier à des connaissances correctes (à partir des savoirs de référence) ou incorrectes (à partir des savoirs des élèves),

Notre point de vue a été de concevoir un diagnostic qui joue un double rôle : renvoyer à l'enseignant, modélisé ici sous la forme d'un conseiller, une image du travail de l'élève, mais aussi renvoyer à l'élève, une image de son propre travail.

\subsection{Méthode}

En ce qui concerne SCHNAPS, nous avons fait l'hypothèse qu'il existe des comportements erronés identifiables, et qu'il est possible de les retrouver dans les actions permises par l'interface. La méthode que nous avons adoptée est la suivante : 1. Recueillir des informations sur les résolutions et les erreurs par l'analyse de copies et de résolutions orales, et les organiser dans une classification descriptive globale,

2. Rechercher une représentation efficace (pour éviter la combinatoire), qui soit orientée vers l'intervention (assez proche de ce qui est dans la résolution de l'élève), et suffisamment générale (pour limiter le nombre des erreurs décrites). 
3. Développer une technique de diagnostic qui s'appuie sur cette représentation et qui s'inspire de la classification heuristique.

L'analyse de résolutions écrites et des protocoles de résolution orales a été effectuée sur le même corpus que celui initialement constitué pour la conception de l'interface de résolution. Elle a été complétée par une observation systématique des résolutions sur machine. En regroupant les difficultés observées en quatre catégories (1 - représentation du problème, 2 - outils et connaissances de base dans les domaines numérique, lexical et chimique, 3 - grandeurs physico-chimiques, 4 relations associées à l'équation-bilan), nous avons observé que la majeure partie de celles qui se retrouvent dans les résolutions sur machine portent sur les grandeurs et les relations qui s'y appliquent.

En adoptant une méthode de classification voisine de celles employées par Noël et Py [NOE 93] en géométrie, ou par Veillette [VEI 91] en thermodynamique, nous avons choisi de représenter les principales erreurs représentatives de ces difficultés en les intégrant dans la base de formules employée pour la résolution par le système.

Le diagnostic que nous avons développé [BLO 95] porte sur la reconnaissance des actions élémentaires au fur et à mesure de la résolution. Parmi celles-ci, les expressions algébriques écrites par l'élève avec la fonction Calcul ou Relation sont celles qui posent de réelles difficultés, les autres fonctions n'offrant qu'un nombre restreint et aisément identifiable de possibilités de déclaration de faits ou de valeurs.

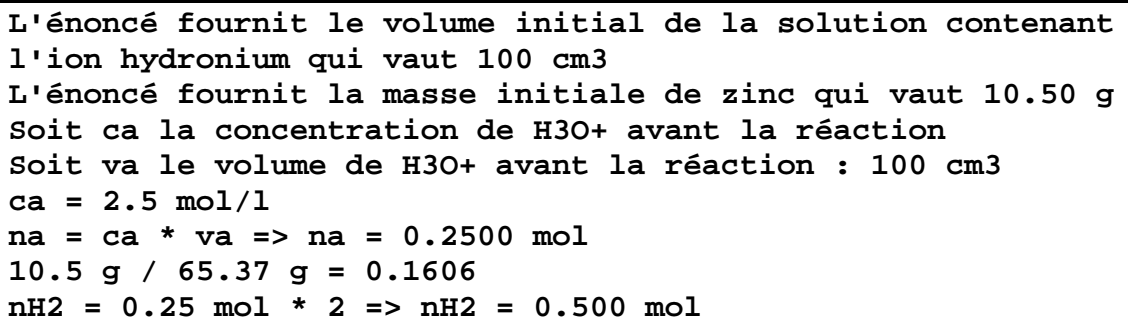

Figure 14. Extrait de rédaction comportant des expressions à diagnostiquer (trois dernières lignes)

\subsection{Base de formules}

Dans la base, chaque formule correcte ou incorrecte est décrite comme l'application d'une formule générale qui correspond à l'écriture sous forme canonique de l'expression la plus générique d'un ensemble de formules. 
Exemple :

(forme

(= (* (Quantite ?Corps1 ?Instant1) (Coefficient ?Corps1))

(* (Quantite ?Corps2 ?Instant2) (Coefficient ?Corps2))))

Une application est décrite par deux ensembles :

- des conditions d'application de la formule générale, conditions indispensables ou facultatives. Ces conditions sont constituées de listes de prédicats dont les arguments sont les variables présentes dans l'expression canonique de la formule.

Exemple :

(cond-min (Differents ?Corps1 ?Corps2)

(Reagit ?Instant 1)

(Reagit ?Instant2))

- des attributs qui sont des symboles (Correct, Incorrect, Intra, Inter, Calcul, Algebre, Conservation,... permettant une indexation de la formule pour l'appariement ou la construction d'une intervention.

Exemple :

(attr Incorrect Inter J.2.1 Algebre)

Une formule générale exprime le plus souvent une relation générique entre grandeurs (par exemple la conservation de la masse) et ses applications indiquent comment peut s'instancier cette relation dans différents types de situations. Une formule peut être considérée comme incorrecte par sa formule générale, ou par l'application de cette formule générale.

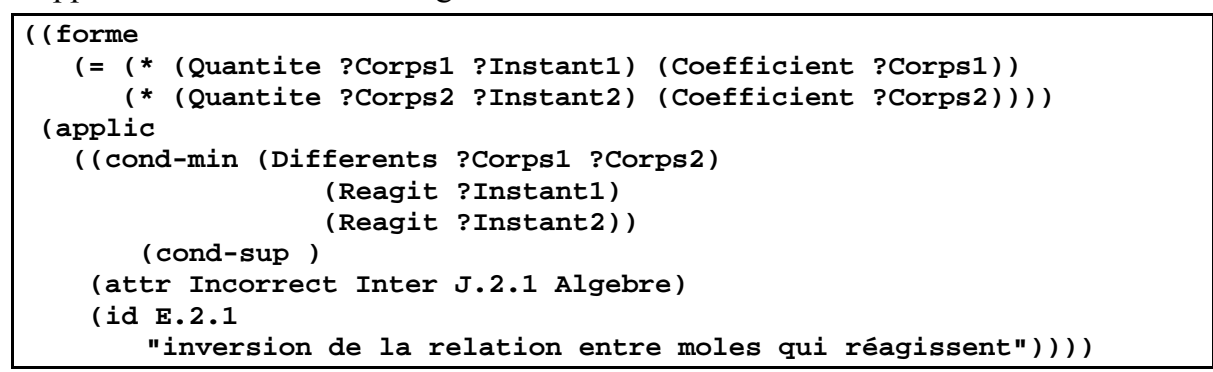

Figure 15. Exemple de formule erronée avec une de ses applications

La base de formules contient 37 formules générales réparties en deux groupes. Dans le groupe composé à partir des relations entre corps différents qui expriment la conservation dans l'équation-bilan, on trouve 6 formules correctes et 6 formules incorrectes, qui comportent au total 28 applications. Dans le groupe composé à partir des relations qui ne portent que sur un seul corps, on trouve 6 formules correctes et 17 formules incorrectes, qui comportent au total 23 applications.

\subsection{Fonctionnement du diagnostic}

La description des classes de comportements par des applications de formules, associée à des critères liés à la forme ou au contexte d'apparition, peut alors être mise en œuvre dans un diagnostic de type classification heuristique [CLA 85]. Cette 
méthode de diagnostic qui permet de prendre en compte les connaissances tirées de l'analyse initiale, comporte essentiellement trois phases : l'abstraction des données, l'association heuristique, et la spécialisation.

L'abstraction des données est ici effectuée par la reconnaissance des opérandes ; il s'agit en effet de passer des valeurs et des variables utilisées par l'élève aux grandeurs identifiables dans le problème.

L'association heuristique correspond à la reconnaissance d'une formule ; l'appariement qui tient compte de la forme algébrique de l'expression et des hypothèses sur les opérandes suggère certaines formules.

La spécialisation consiste alors à retenir la (ou les) formule(s) qui représente(nt) au mieux ce que l'élève a écrit en tenant compte des indices complémentaires comme le caractère plausible des instanciations des parties variables de la formule.

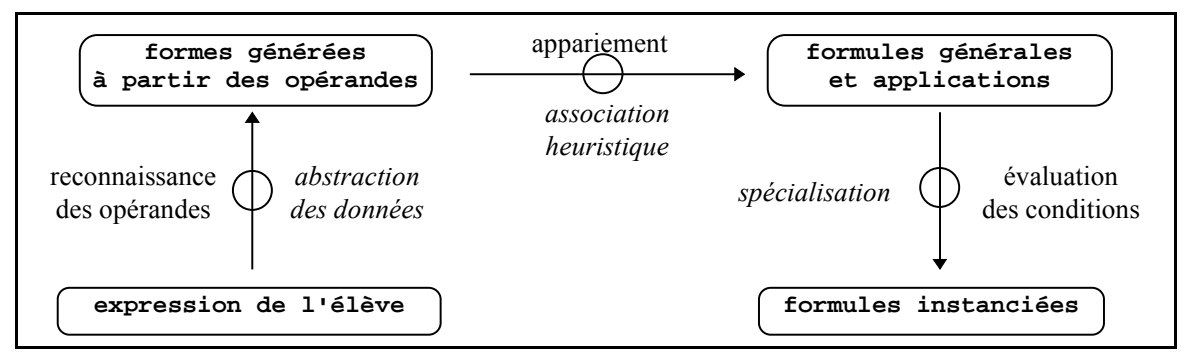

Figure 16. La classification heuristique mise en ouvre dans le diagnostic

Le diagnostic se déroule donc en trois étapes principales : la reconnaissance des opérandes, l'appariement avec la base de formules, et l'évaluation des conditions d'application. Ces étapes sont itérées jusqu'à ce que les résultats obtenus répondent à un ensemble de critères de terminaison.

Les critères de terminaison portent sur la durée effective de la recherche (pour pouvoir intervenir dans un délai minimum), sur la fiabilité des hypothèses effectuées sur les opérandes (les hypothèses sont classées en fonction de l'origine supposée des opérandes) et sur le nombre des formules analysées (toutes les formules correspondant au même jeu d'hypothèses sont analysées).

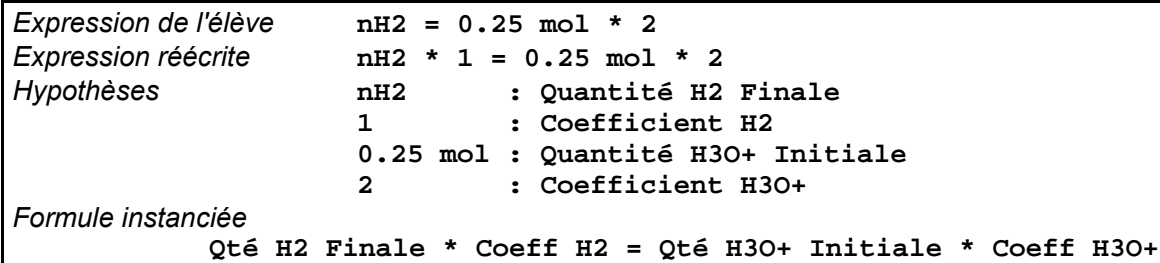

Figure 17. Exemple de résultat simplifié du diagnostic d'une expression

En général, le diagnostic ne fournit pas un résultat unique mais un ensemble de résultats, à savoir : plusieurs formules générales, une ou plusieurs applications de ces formules, et des hypothèses sur les opérandes pour chaque application. Ceux-ci sont ordonnés pour en extraire le plus probable et le plus favorable à l'élève. 


\section{Aides et interventions directes}

\subsection{Introduction}

Au delà de l'aide indirecte apportée par les outils de résolution qui facilitent la décomposition de la tâche et la structuration de la rédaction, se pose la question de déterminer quelles aides explicites intégrer à l'environnement et quelle peut être la part d'initiative du système dans la présentation de ces aides.

Bien que les interventions du système puissent venir perturber le raisonnement de l'élève, ce qui a incité certains concepteurs à ne proposer que des aides passives [MOB 92], la situation d'apprentissage autonome incite cependant à développer de telles interventions, tout en cherchant à ne pas s'écarter du but poursuivi par l'élève.

Afin de dépasser le caractère insuffisant des aides fonctionnelles qui se limitent le plus souvent à une présentation détaillée des outils et ne répondent qu'à une petite partie des questions posées par les utilisateurs, plusieurs systèmes d'aide associés à des environnements d'apprentissage ont été proposés :

- les hypertextes conceptuels (AUTO-DIDACT [MAS 96], HIPOCAMPE [WEN 95]) organisent la présentation des concepts du domaine en un réseau accessible par un hypertexte, la question principale étant de déterminer quelle entrée et quel parcours suggérer à l'élève dans cet hypertexte,

- des systèmes qui prodiguent des conseils en gérant un dialogue avec l'élève (Unix Consultant [CHI 91], EuROHElP [WIN 92], Studia [CHE 94], Disco [LEN 95]),

- des systèmes de conseils plus spécialisés dans l'aide à la tâche, venant le cas échéant se greffer sur l'application sans la perturber (EPITALK [PAQ 94]),

- des agents qui analysent l'activité de l'élève avec précision et qui anticipent les demandes d'aide éventuelles (COACH [SEL 94]).

Pour la conception de l'aide, nous nous sommes inspirés de ces systèmes qui nous semblaient répondre, au moins partiellement, aux attentes des utilisateurs telles que nous les avions perçues lors des premières expérimentations de SCHNAPS.

\subsection{Méthode}

Pour mieux cerner ces attentes, nous avons entrepris une analyse des demandes d'aide provenant des élèves et des interventions des enseignants en situation de tutorat.

Notre objectif principal était de recueillir des données qualitatives sur les demandes d'aide et la nature des interventions d'un enseignant placé dans une situation de tuteur. La méthode que nous avons adoptée consistait à observer l'interaction directe entre un enseignant et un élève qui résout des problèmes avec le prototype SCHNAPS dans lequel aucun dispositif d'aide n'était fonctionnel. Les enregistrements et les traces de quatre séances de travail (deux par élève) ont ainsi été recueillis et analysés.

\begin{tabular}{|l|l|}
\hline Demandes portant sur le & "Comment faire pour écrire la masse molaire \\
\hline
\end{tabular} 


\begin{tabular}{|l|l|}
\hline fonctionnement du logiciel & sur la feuille de rédaction ?" \\
\hline $\begin{array}{l}\text { Demandes portant sur des faits } \\
\text { ou des notions }\end{array}$ & $\begin{array}{l}\text { "Il me faudrait la formule de la poudre noire ... } \\
\text { parce qu'on me dit que c'est un mélange... " }\end{array}$ \\
\hline Demandes d'explications & $\begin{array}{l}\text { "Je me demande pourquoi il ne trouve pas pareil } \\
\text { puisque c'est lui qui a calculé ?" }\end{array}$ \\
\hline Demandes d'évaluation & $\begin{array}{l}\text { "Est-ce que je peux vérifier ça avant de } \\
\text { continuer ?" }\end{array}$ \\
\hline $\begin{array}{l}\text { Interrogations sur ce que sait le } \\
\text { système }\end{array}$ & "Tout çà, il sait ce que c'est?" \\
\hline
\end{tabular}

Figure 18. Les demandes d'aide

Des demandes d'aide explicites et implicites ont été relevées en assez grand nombre alors que les interventions de l'enseignant qui ne répondaient pas directement à une demande ont été moins fréquentes.

\begin{tabular}{|l|l|}
\hline $\begin{array}{l}\text { Interventions portant sur le } \\
\text { fonctionnement du logiciel }\end{array}$ & $\begin{array}{l}\text { "Attention, si tu veux qu'elle calcule y, il faut que } \\
\text { tu écrives l'expression dans le sens y=... " } \\
\text { "Chaque fois que tu voudras écrire une relation } \\
\text { ou faire un calcul, tu passes par l'option Calcul } \\
\text { ".. }\end{array}$ \\
\hline $\begin{array}{l}\text { Interventions portant sur les } \\
\text { notions }\end{array}$ & $\begin{array}{l}\text { "Une quantité de matière, c'est un nombre de } \\
\text { moles, et en fait mole c'est une unité." }\end{array}$ \\
\hline $\begin{array}{l}\text { Interventions visant à expliquer } \\
\text { un message ou un résultat }\end{array}$ & $\begin{array}{l}\text { "... tu avais demandé un calcul et là tu en } \\
\text { demandes un autre } \text { il y avait Égalité incorrecte } \\
\text { parce que tu demandes de calculer la même } \\
\text { chose avec deux résultats différents" }\end{array}$ \\
\hline Conseils sur l'emploi des outils & $\begin{array}{l}\text { "Tu devrais utiliser Définition et donner un nom } \\
\text { au volume molaire }\end{array}$ \\
\hline
\end{tabular}

Figure 19. Les interventions de l'enseignant

Ces résultats montrent l'importance des questions des élèves portant sur les outils, les messages, le fonctionnement du logiciel, sur les notions et sur les méthodes de résolution. Ils font apparaître quelques caractéristiques plus inattendues : les questions que se posent les élèves sur ce que le système connaît, et les nombreux conseils d'ordre général donnés par l'enseignant.

Une analyse plus quantitative de K. Van Lehn qui a porté sur des demandes d'aide pendant la résolution de problèmes de mécanique [VAN 96] révèle qu'une majorité relative des demandes (24 sur 73) proviennent d'un blocage ("Que faire maintenant ?") et que les autres sont des demandes d'évaluation ("Est-ce correct ?"), des questions sur les méthodes ("Comment faire pour ... ?") et des incompréhensions révélatrices de conceptions erronées.

Les interventions de l'enseignant qui ne répondent pas à une demande d'aide explicite portent essentiellement sur les mêmes contenus que les demandes des 
élèves. Ces interventions "spontanées" sont peu nombreuses. On y trouve peu d'explications sur les méthodes de résolution, confirmant ainsi une attitude générale qui consiste à en dire le moins possible. En revanche, elles comportent bon nombre de conseils sur la "bonne" manière d'utiliser ce qui est disponible. Enfin, nombre de ces interventions prennent une forme interrogative visant à faire expliciter par l'élève une écriture ou une action imprécise ou ambiguë.

Les résultats de cette analyse incitent à rechercher une organisation de l'aide qui puisse prendre en compte ces différents aspects.

\subsection{Assistants}

Afin de pouvoir répondre de manière différenciée aux différentes demandes explicites ou implicites, l'aide proposée dans SCHNAPS est décomposée en ensembles homogènes dénommés assistants. Les assistants sont des entités indépendantes et spécialisées qui répondent à des sollicitations : demandes de l'utilisateur ou interrogations du système pour effectuer une intervention. Il s'agit de rassembler au sein d'une même entité tout ce qui concourt à un type d'aide précis.

Un assistant est chargé de toutes les aides qui sont reliées par une unité thématique, stylistique ou pédagogique. Il vise à apporter une aide dans un but déterminé. Il est caractérisé par :

- Un contenu :

Ce contenu est constitué de messages correspondant aux plus petites entités qui peuvent être présentées à l'utilisateur. Les messages sont principalement des textes et des graphiques, soit fixes, soit paramétrables.

- Une logique propre :

Cette logique de comportement vise à conserver une cohérence des réponses de l'assistant, en accord avec le but qui lui a été fixé. Il s'agit principalement de sélectionner les messages potentiels en fonction de la sollicitation et de l'activité de l'élève, en tenant compte des paramètres généraux de l'aide, et en respectant un certain nombre de principes d'intervention, comme ceux énoncés dans [DEL 92] [GRI 75] [WIN 92].

- Un mode de présentation des messages :

Cette présentation peut varier suivant la taille des messages mais aussi en fonction de l'intégration de l'assistant dans l'activité de résolution. Ainsi, les messages sont présentés dans une fenêtre autonome pour les concepts, ou dans une zone intégrée à la fenêtre de recherche/rédaction pour les conseils.

Le contrôle de l'aide apportée à l'élève est réparti entre deux niveaux ; au niveau général, il porte sur le choix d'un assistant et la décision d'intervenir, et, au niveau particulier de chaque assistant, sur l'opportunité et le choix du message présenté.

\subsection{Caractéristiques et contrôle des assistants}

Les assistants qui répondent à une demande requièrent des messages plus importants et plus élaborés. Ils se caractérisent par l'analyse de la demande et par la 
gestion de l'accès à l'information. Nous en avons proposé trois : une aide sur les outils, une aide conceptuelle, et une aide sur les méthodes.

Les assistants qui correspondent à des interventions du système utilisent des messages peu nombreux et plus courts. Ils se caractérisent par une analyse de l'activité issue du diagnostic et par le choix du message et la génération de ces composantes. Nous avons proposé quatre assistants de ce type : des interventions pour préciser la rédaction, des interventions spécifiques sur les concepts, des conseils de rédaction, et des interventions générales.

L'activation d'une intervention qui vient perturber le déroulement normal du travail, suppose de prendre en considération de nombreux paramètres. Dans un premier temps, nous avons choisi de retenir deux éléments déterminants : le degré d'intervention et la phase pédagogique, auxquels s'ajoutent l'historique de l'aide, c'est-à-dire la nature des interventions précédentes et la durée entre ces interventions.

Le degré d'intervention, fixé par l'enseignant, permet de déterminer la fréquence des interventions par rapport aux actions effectuées par l'élève.

Le découpage de la séquence de travail en plusieurs phases pédagogiques distinctes a été mis en évidence dans l'analyse de dialogues entre un professeur et un élève en difficulté [CAU 92]. Nous avons identifié quatre phases à l'intérieur de la résolution du même problème: une phase de définition et d'appropriation du problème, une phase d'exploration qui transforme les données initiales par des relations entre grandeurs du même corps, une phase de résolution mettant en oeuvre diverses stratégies incluant une ou plusieurs relations-clés, et une phase de bilan, à la fin de la résolution décidée par l'élève.

Le passage d'une phase à la suivante est déterminé d'après le type des actions effectuées, leur nombre, les attributs des formules reconnues, le temps écoulé depuis le début de la session.

Chaque phase pédagogique privilégie un ou plusieurs types d'intervention : les suggestions d'aide au cours de la phase de définition, les interventions pour préciser la rédaction au cours de la phase d'exploration, les conseils de rédaction et les interventions sur les concepts au cours de la phase de résolution.

\subsection{Description des principaux assistants}

\subsubsection{Aide conceptuelle}

L'aide conceptuelle répond à deux objectifs complémentaires : le premier est un rappel de connaissances considérées comme connues, le second étend ces connaissances essentielles en apportant des détails et des précisions ou en apportant de nouvelles connaissances, à l'instar de ce que propose Van Lehn dans l'apprentissage de concepts en physique [VAN 96].

L'ensemble des concepts que nous avons retenus recouvre les entités qui interviennent dans la représentation et la résolution du problème et aussi les notions qui expliquent cette représentation. Les concepts sont organisés par un seul type de lien (généralisation/spécialisation) formant ainsi une hiérarchie. 
Le contenu de l'aide associée à un concept comprend :

- un terme qui sert à la fois pour afficher le concept et pour y accéder, et qui représente l'aspect terminologique du concept,

- un texte essentiel, qui correspond le plus souvent à une définition du concept,

- des compléments qui relèvent de deux types : des compléments explicatifs, exemples qui permettent de moduler et de préciser le texte essentiel, des compléments conceptuels, associant un ou plusieurs autres concepts au concept de référence et permettant de montrer les relations entre ces concepts. Les compléments peuvent comporter des textes et des graphiques interactifs, dont certains sont inspirés des schémas relationnels proposés dans [MOR 92].

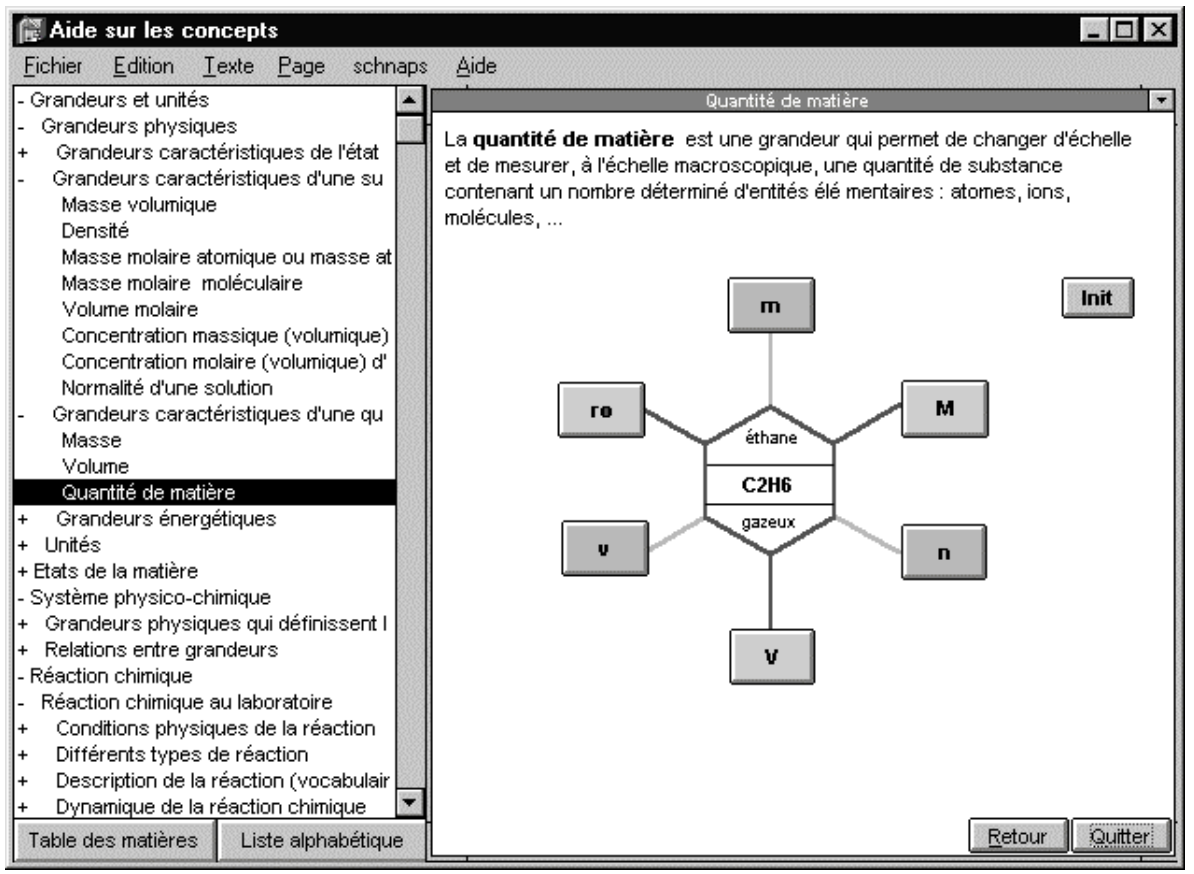

Figure 20. Aide sur le concept de quantité de matière, comportant texte et graphique interactif

Cette aide est accessible par un menu de la fenêtre de définition ou de rédaction. La réponse à une demande d'aide sur un concept est constituée du texte essentiel et des compléments choisis en fonction de quelques principes.

L'idée principale est de fournir l'information par accumulation, en ajoutant les éléments qui semblent intéresser l'élève ou lui poser des difficultés. Ainsi, un complément explicatif est ajouté si l'élève est débutant ou si des erreurs ont été constatées sur ce concept. Un complément sur un autre concept est ajouté si l'aide portant sur cet autre concept a déjà été demandée, ou si une erreur concernant cet autre concept a déjà été observée. 
Cet assistant peut aussi fonctionner en mode autonome, indépendamment de l'environnement SCHNAPS.

\subsubsection{Aide sur les méthodes}

Ce type d'aide est destiné à répondre à la question générale souvent posée par les élèves sous la forme: "Comment faire pour résoudre le problème ?" ou "Que faire ...?"

A ces questions trop générales, l'assistant propose des réponses partielles qui exposent des méthodes de résolution décrites par les plans utilisés dans le résolveur. Ces plans ont en effet été conçus pour décrire les méthodes habituelles utilisées par les élèves ou préconisées par les enseignants.

La présentation des plans nécessite une description textuelle des objectifs, des conditions d'application, et des procédures associées aux plans. Cette présentation est construite à partir de la définition du plan et des descriptions associées à chacun de ses éléments. L'entrée principale se fait par un objectif. Les actions du plan sont affichées en indiquant suivant les cas si leur application est fixe et sans conditions ("Faire dans l'ordre") ou si elle dépend de conditions ("Suivant les cas") ou si leur résultat est inconnu ("Essayer").

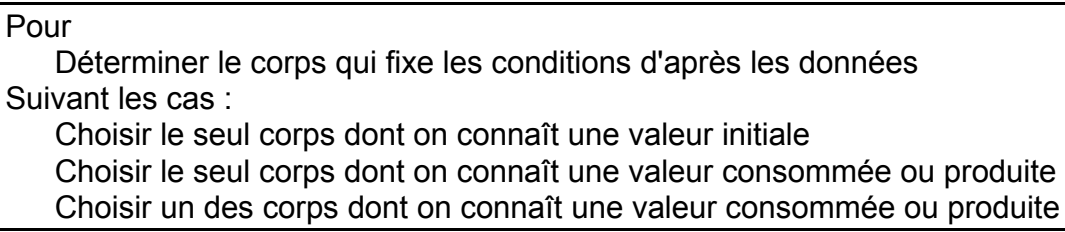

Choisir le seul corps dont on connaît une valeur initiale

Choisir le seul corps dont on connaît une valeur consommée ou produite

Choisir un des corps dont on connaît une valeur consommée ou produite

Figure 21. Présentation du plan FIXER-C2

Le mode d'interrogation des méthodes permet d'explorer les plans de résolution à partir de la question initiale en ne développant qu'un seul niveau à la fois. L'élève parcourt l'arbre des plans niveau par niveau. Il obtient ainsi une vision hiérarchisée des actions possibles qui met l'accent sur la décomposition des actions.

\subsubsection{Conseils de rédaction}

Ces interventions ont pour but d'améliorer la manière dont l'élève utilise les outils et portent essentiellement sur le style de la rédaction.

Les conseils présentés sont inspirés par un souci de clarté et d'efficacité de la rédaction. Ils correspondent en général à des conseils qui sont donnés par les enseignants pour la rédaction sur papier. Assez peu nombreux, ils sont constitués de textes prédéfinis, courts, ne portant que sur un seul sujet à la fois.

Vous devriez définir au préalable les variables que vous utilisez dans vos calculs et vos relations.

Figure 22. Exemple de conseil de rédaction

Pour un instant donné de la résolution, les conseils candidats sont déterminés par des règles dont les conditions analysent certains éléments fournis par le diagnostic 
des expressions, comme par exemple l'emploi des unités, et par une analyse globale de l'usage des différents outils depuis le début de la session. Les conseils candidats sont ensuite classés par un ordre de pertinence fixé a priori. Chaque conseil ne peut être émis qu'une seule fois au cours de la même session.

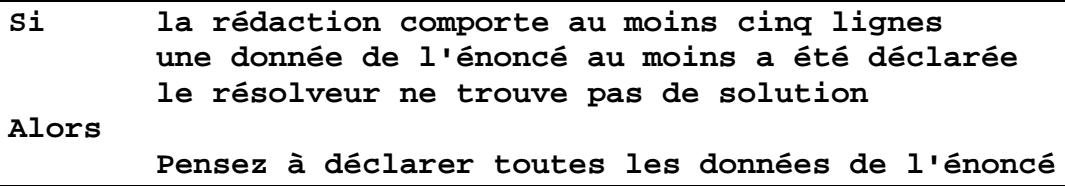

Figure 23. Exemple de règle de détermination d'un conseil de rédaction

\subsubsection{Interventions pour préciser la rédaction}

Ces interventions ont pour origine des imprécisions dans l'écriture de la résolution par l'élève et sont utiles pour affiner le résultat du diagnostic.

On pourrait considérer a priori qu'elles sont destinées à un usage interne du système ou à l'usage de l'enseignant. Nous pensons cependant que ces interventions constituent une aide importante, bien qu'elle soit souvent indirecte, car elles attirent l'attention de l'élève sur un élément particulier de sa rédaction. De plus, elles l'encouragent à analyser sa propre activité et à mieux se l'expliquer, mettant en pratique une sorte d'auto-explication (self-explanation) dont l'efficacité a été mise en évidence en matière d'apprentissage [CHI 89].

Le contenu de ces interventions est constitué de messages paramétrés par des éléments de la rédaction. Pour prendre en compte des formes d'interaction qui n'ont pas toutes le même impact sur l'élève, nous utilisons deux types principaux d'intervention : confirmation et interrogation.

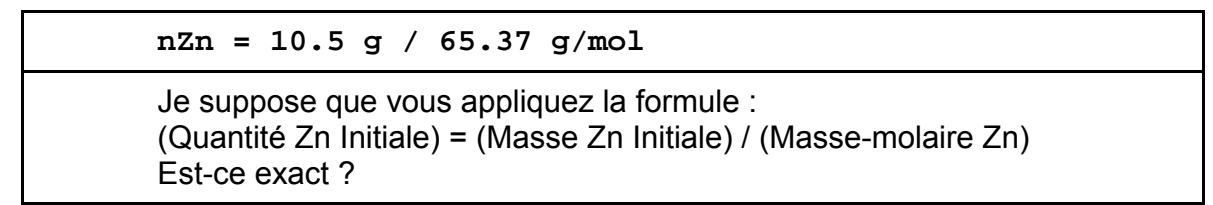

Figure 24. Expression de l'élève et message d'intervention

Les interventions possibles sont déterminées par des règles qui s'appuient sur les résultats du diagnostic : type de l'expression, hypothèses sur les opérandes, hypothèses sur les formules, formules justes ou erronées. Le choix de la meilleure intervention est déterminé d'après une valeur d'intérêt du message, attribuée a priori lors de la rédaction du message. 


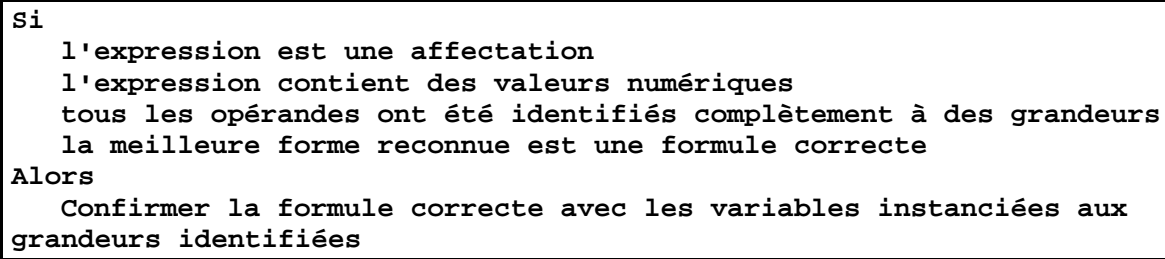

Figure 25. Exemple de règle de détermination d'une intervention

\subsubsection{Interventions spécifiques sur les concepts}

Ces interventions sont destinées à rappeler des informations sur les notions qui semblent faire difficulté. Il ne s'agit pas de corriger l'élève, ce qui n'est pas toujours efficace, mais de porter son attention sur un ou deux points qui semblent expliquer la difficulté. L'origine de ces difficultés est tirée des erreurs associées aux formules reconnues par le diagnostic.

Pour déterminer le contenu de ces interventions, cet assistant utilise d'une part les hypothèses du diagnostic sur les erreurs contenues dans l'expression, et d'autre part les principaux concepts qui ont été associés à ces hypothèses.

Les contenus de ces interventions sont de deux ordres :

- Le premier est en liaison directe avec l'erreur reconnue et porte sur le caractère de cette erreur (substitution de deux grandeurs, relation erronée, transformation algébrique). A chaque caractère est associé un texte qui détaille les éléments supposés de l'erreur.

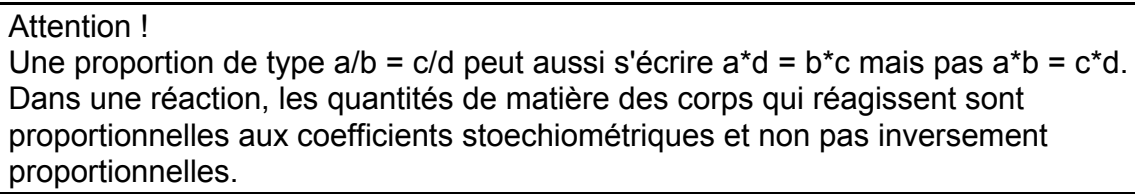

Figure 26. Message associé à une inversion de la proportion entre quantités de matière

- Le second est plus indirect et porte sur les concepts que l'on peut associer à la formule reconnue. Dans ce deuxième cas, le contenu de l'intervention est un texte tiré des aides associées aux concepts.

L'association entre les erreurs et les caractères et les concepts est définie dans la base de formules. A chaque formule erronée sont associés, en plus de la formule juste correspondante quand elle existe, un identifiant du caractère de l'erreur, et une liste de concepts dont on fait l'hypothèse qu'ils expliquent en partie l'erreur en question.

Ces messages sont associés non seulement aux formules erronées mais aussi aux conditions d'application des formules correctes : instants différents, corps différents, corps non gazeux, corps non limitant, etc. Comme les interventions pour préciser la rédaction, les interventions possibles sont déterminées par des règles qui s'appuient sur les résultats du diagnostic. 


\subsubsection{Interventions générales}

Ce type d'interventions que nous pouvons qualifier de méta-interventions est destiné à anticiper des questions que se posent les élèves sur le système lui-même.

Ces interventions, portant sur les aides et sur les interventions elles-mêmes, cherchent à expliquer quels sont les objectifs du système, ce qui est attendu de l'élève, comment il peut travailler au mieux avec l'environnement, ce que l'élève peut attendre du système, et en particulier quelles aides il peut obtenir et quelles interventions peuvent survenir.

Ces interventions, en nombre limité, contiennent des conseils de travail très généraux, des suggestions d'appel de l'aide, des suggestions pour commencer, des explications à la fin de la session.

La détermination de ces interventions est semblable à celle qui régit les conseils de rédaction. Elle dépend de l'analyse du style de la résolution et de l'analyse des conseils déjà donnés. Ces interventions sont toujours possibles quelle que soit la phase pédagogique, mais leur contenu en dépend.

Essayez de commencer en utilisant tout ce que vous connaissez. Vous pourrez alors bénéficier de conseils plus précis et plus efficaces.

Figure 27. Exemple d'intervention générale déclenchable en début de session

\section{Réalisation et expérimentation}

\subsection{Prototype initial}

Le prototype initial de SCHNAPS a été développé intégralement en langage SCHEME (dialecte LisP à liaison lexicale) pour PC sous système MSDOS. Il comprend le résolveur, les outils de calcul avec unités (la calculette chimique), l'interface de définition, l'interface de recherche et de rédaction, et la base de données chimiques.

Cette version minimale a été expérimentée auprès de 9 classes de lycée (7 Secondes et 2 Premières).

L'examen de l'utilisation des fonctions de recherche et de rédaction met en évidence de grandes variations entre les individus.

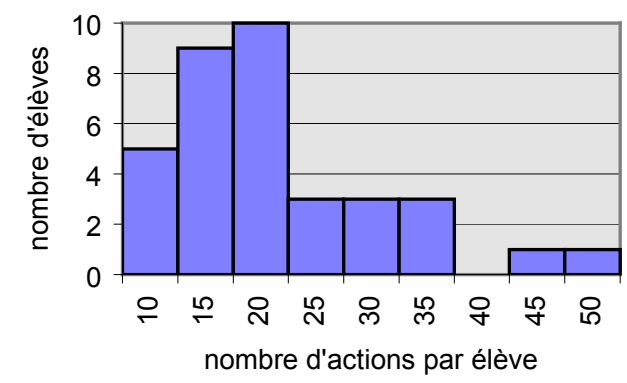

Figure 28. Histogramme du nombre d'actions (classe de Seconde, première séance) 
La durée de travail y est nettement plus importante que pour une résolution habituelle. Cette augmentation de $30 \%$ à $50 \%$ du temps peut s'expliquer par la lenteur due à la dactylographie et par un souci de qualité de la rédaction manifesté par la majeure partie des élèves.

Cette augmentation de la durée est appréciée différemment suivant les individus. Alors que les élèves qui maîtrisent correctement ces problèmes ne voient pas l'intérêt de l'outil, ce qui est compréhensible, une majorité estime que cet allongement est compensé par d'autres avantages ("nécessité d'être attentif", "permet de réfléchir", "fait les calculs", etc.). Pour les élèves qui éprouvent des difficultés dans la résolution, il n'est pas apparu que le temps de travail nécessaire pour aboutir à un résultat identique en termes de contenu et de qualité soit sensiblement supérieur.

Le nombre d'actions et en particulier d'expressions incorrectes est sensiblement inférieur à celui que l'on peut observer sur des rédactions habituelles sur papier. Ceci est en partie expliqué par l'élimination de la majorité des erreurs numériques et des erreurs dimensionnelles grâce au calcul avec unités. D'autre part, la rigueur syntaxique imposée par les outils réduit la part d'imprécision des rédactions.

La métaphore de la feuille de brouillon que nous avons utilisée pour définir l'interface de recherche n'a pas été immédiatement perçue par les élèves. Plusieurs indices permettent de penser que ceux-ci ont privilégié la rédaction par rapport à la recherche de la solution. Cependant, le recours plus régulier aux outils de calcul s'est amplifié au cours des séances. L'image d'un résolveur automatique, initialement prépondérante, s'est estompée au fur et à mesure de l'utilisation.

Enfin, nous avons pu constater que, même en l'absence de module spécifique d'aide, le logiciel est vu par de nombreux élèves comme une aide indirecte et comme une incitation à rédiger correctement.

\subsection{Prototype avec diagnostic}

Le diagnostic, développé avec les mêmes outils, a été testé sur un grand nombre de traces enregistrées au cours des expérimentations précédentes.

En analysant avec précision les résultats obtenus sur une dizaine de sessions, on peut estimer que les $2 / 3$ des expressions algébriques sont interprétées sans ambiguïté et de manière satisfaisante. Parmi les cas qui restent (reconnaissance incertaine qui retient plus d'une formule et reconnaissance impossible), les détails de l'analyse fournissent des informations suffisantes sur les hypothèses en présence ou sur les éléments non reconnus pour permettre des interventions adaptées.

A la suite de ces tests, nous nous sommes rendus compte que l'un des problèmes les plus délicats à l'issue du diagnostic est de décider si ce que l'élève a écrit est correct ou acceptable dans le contexte de la résolution. Ceci suppose une très bonne efficacité de cet aspect du diagnostic, c'est-à-dire une complétude de la base de formules correctes, l'application de toutes les transformations algébriques possibles et une évaluation fiable de la qualité de la reconnaissance. Nous pouvons estimer que notre prototype s'avère efficace, sauf en ce qui concerne les transformations 
algébriques. La reconnaissance des formules incorrectes est moins fréquente et moins fondamentale, bien qu'elle permette des interventions beaucoup plus ciblées.

Les échecs du diagnostic ont des causes variées : la simplification numérique (tous les cas de simplification ne sont pas pris en compte dans la version utilisée), la combinaison de formules, les formules aberrantes (absentes de la base de formules), l'impossibilité de reconnaître des opérandes, la durée du diagnostic. En revanche, nous n'avons constaté que très peu d'erreurs d'analyse (une formule ou une grandeur mal interprétée), car le diagnostic privilégie ce qui est correct, au risque d'attribuer à l'expression de l'élève une interprétation qui n'est pas toujours prouvée.

\subsection{Version complète}

La version complète est une refonte complète de la version précédente effectuée avec des outils de développement différents pour l'interface et pour le noyau du système. Les interfaces sont développées avec TOOLBOOK, ensemble d'outils et de fonctions de présentation qui permettent une intégration de graphique et de texte avec des fonctionnalités hypertexte. Le noyau est développé avec SCHEME-TO-C, dans un environnement de programmation que nous avons développé pour WINDOWs à partir d'une version distribuée par DEC pour plusieurs plates-formes et qui permet à la fois l'interprétation et la compilation de fonctions écrites en SCHEME. Les deux applications communiquent par messages au moyen de la procédure d'échange dynamique de données de WINDOWs.

Les premiers essais de cette version avec quelques élèves ont confirmé les résultats précédents (nécessité de "tout expliquer" et intérêt de détailler les étapes de la résolution) et l'utilité des outils de rédaction (pour indiquer un sous-but et pour introduire une relation).

Les interventions du système sont apparues bien adaptées à la situation mais n'ont pas toujours été bien prises en compte. Les appels à l'aide explicite ont révélé, comme on pouvait s'y attendre, une difficulté des élèves à formuler et à trouver précisément ce qu'ils voudraient.

Enfin, la richesse de l'ensemble provoque lors des premières sessions une certaine désorientation des élèves face à un environnement de travail dont ils ne connaissent pas d'équivalent.

Ces premiers essais confirment la nécessité d'une évaluation complète des environnements interactifs d'apprentissage dans leur contexte d'utilisation prévu, au cours du cycle de conception.

\section{Conclusion}

Les outils de définition et surtout de résolution ont été très précieux pour analyser l'activité de l'élève. En incitant à détailler et à expliciter certaines étapes de la résolution, ils s'avèrent apporter une aide indirecte à la catégorie d'élèves à laquelle ce type d'environnement s'adresse, élèves disposant des connaissances de base mais éprouvant des difficultés de mise en oeuvre. 
La conception et l'expérimentation de ces outils ont remis en avant quelques questions, à la frontière de la didactique, de l'informatique et de l'ergonomie cognitive [SCH 96]: quel langage permet de représenter le système physicochimique ? comment représenter l'aspect dynamique de la réaction? quel espace de connaissances est nécessaire pour traiter un problème de ce type ? quelle est la nature de la complexité de ces problèmes pour les élèves?

La description des principales erreurs par des formules et leurs applications, associée à une méthode de classification heuristique, a montré son efficacité dans l'analyse d'une grande partie de l'activité de l'élève. Elle fournit des résultats suffisamment détaillés pour générer des interventions et favoriser ainsi de la part de l'élève une analyse réflexive sur sa propre activité.

Une extension du diagnostic par la reconnaissance de plans dans la rédaction de l'élève reste un problème difficile dans la mesure où les élèves emploient peu de méthodes explicites. La possibilité de déclarer un but intermédiaire est une solution pour pallier cette difficulté. La mise au point d'un diagnostic complet nécessiterait de revoir l'analyse de la tâche de résolution et de caractériser de manière plus approfondie les modèles de résolution des élèves [BEN 95].

La décomposition de l'aide en assistants indépendants a grandement facilité la tâche de conception des aides et des interventions. Elle permet une meilleure adaptation au moment de la conception et un réglage plus faciles de la part de l'élève ou de l'enseignant au moment de l'utilisation. Certains assistants relativement indépendants de l'environnement peuvent être réutilisés sans adaptation supplémentaire dans un autre contexte ; c'est le cas de l'aide sur les concepts ou de l'aide sur les méthodes.

Les principes qui ont prévalu à la conception des assistants qui prennent l'initiative (influence du temps, homogénéité des assistants en fonction des objectifs visés, forme des messages) sont généralisables à d'autres situations d'aide directe. Une expérimentation de ce système d'aide à plus grande échelle est cependant nécessaire pour préciser et confirmer ces idées, que nous comptons reprendre pour concevoir des agents capables d'assister un élève dans d'autres situations d'autoformation en sciences.

\section{Bibliographie}

[BAL 94] Balacheff N., Didactique et Intelligence Artificielle, in Didactique et Intelligence Artificielle, Balacheff \& Vivet (eds), La Pensée Sauvage, 1994, 9-42.

[BEN 95] Bental D., Tiberghien A., Baker M., Megalagaki O., Analyse et modélisation des notions de l'énergie dans l'environnement "CHENE", in Environnements Interactifs d'Apprentissage avec Ordinateur, tome 2, Guin D., Nicaud J.F., Py D. (Eds), Eyrolles, 1995, pp. 137-148.

[BER 95] Bernat P., Morinet-Lambert J., Spécificités et modélisation de l'interaction dans un EIAO, in Environnements Interactifs d'Apprentissage avec Ordinateur, tome 2, Guin D., Nicaud J.F., Py D. (Eds), Eyrolles, 1995, pp. 209-220.

[BIE 92] Bierman D.J., Kamsteg P.A., Sandberg J.A., Students Models, Scratch-pads and Simulation, in New Directions for Intelligent Tutoring Systems, Costa E. (ed), SpringerVerlag, NATO ASI Series, 1992, pp. 135-145. 
[BLO 95] Blondel F.M., Schwob M., Tarizzo M., Diagnostic des actions de l'élève en vue d'une interaction dans un environnement ouvert en chimie, in Environnements Interactifs d'Apprentissage avec Ordinateur, Guin D., Nicaud, J-F. Py D. (eds), Eyrolles, 1995, pp. 149-160.

[BLO 96] Blondel F.M., Diagnostic et aide en EIAO, Etude d'un environnement d'aide à la résolution de problèmes en chimie, thèse de l'Université Henri Poincaré - Nancy I, juillet 1996, $269 \mathrm{p}$.

[BRU 97] Bruillard E., Les machines à enseigner, Hermès, 1997, 320 p.

[CAB 87] Cabrol D., Cachet C. Cornelius R., A problem-solving partner using Prolog, in $A$ Computer or Each Student, R.Lewis and E.D.Tagg (eds), IFIP, Elsevier Science Publishers (North-Holland), 1987, 159- 166.

[CAB 88]Cabrol D., Cachet C., Coopération entre un système de simulation d'expériences et un système expert de conseils pédagogiques, Actes 3èmes Journées Informatique et Pédagogie des Sciences Physiques, INRP-UDP, Grenoble, 1988, 77-82.

[CAB 91] Cabrol D., Luft R., Moore J., Développement de systèmes didactiques ouverts dans un environnement de découverte et d'apprentissage en chimie, in Actes des Journées EIAO de Cachan, Baron M., Gras R. \& Nicaud J.F. (eds), ENS de Cachan, 1991, pp. 141164.

[CAB 94] Cabrol D., Rabine J-P., Ricard D., Rouillard M., Working with IRexpert : an Infrared Interpretation Assistant, in Proceedings of CALISCE'94, Telecom, Paris, 1994, pp. 439-442.

[CLA 85] Clancey W.J. [1985] : Heuristic Classification, Artificial Intelligence, vol 27, pp. 289-350.

[CHE 94] Chevallier R., Gestion de dialogues en EIAO : le système tutoriel STUDIA, Sciences et Techniques Educatives, vol 1, n³, 1994, pp. 373-396.

[CHI 89] Chi M., Bassok M., Lewis M., Reimann P., Glaser R., Self-explanations : How students study and use examples in learning to solve problems, Cognitive Science, vol 15, 1989, pp. 145-182.

[CHI 91] Chin D.N., Intelligent Interfaces as Agents, in Intelligent User Interfaces, Sullivan J. \& Tyler S. (eds), ACM Press, 1991,177-206.

[DEL 92] Delozanne E., Explications en EIAO : Etudes à partir d'ELISE, un logiciel pour s'entraîner à une méthode de calcul des primitives, Thèse de l'Université du Maine, 1992.

[DEL 94] Delozanne E., Un projet pluridisciplinaire : ELISE, un logiciel pour donner des leçons de méthode, Recherches en Didactique des Mathématiques, vol 14, n¹2, 1994, pp. 211-250.

[DES 96] Desmoulins C., Le diagnostic de la correction d'une figure géométrique : le cas du système CALC, Sciences et Techniques Educatives, vol 3, n², 1996, pp. 191-226.

[DIL 94] Dillenbourg P., Evolution épistémologique en EIAO, Sciences et Techniques Educatives, vol 1, n¹, 1994, 39-51.

[DIM 95] Dimitracopoulou A., Le tutorat dans les systèmes informatisés d'apprentissage: Etude de la conception et réalisation d'un tutoriel d'aide à la représentation physique des situations étudiées par la mécanique, thèse de l'Université Paris 7, 1995, 382 p.

[DUM 95] Duma J., Giroire H., Tisseau G., Le Calvez F., Urtasun M., Mise en évidence de styles de résolution, Evolution de l'Interface dans le projet Combien?, in Environnements Interactifs d'Apprentissage avec Ordinateur, tome 2, Guin D., Nicaud J.F., Py D. (Eds), Eyrolles, 1995, pp. 245-255.

[FOR 93] Forte E.N., Herzog J.-M., Wentland M.C., Identification de Concepts et Parcours Orienté dans un Hypertexte Pédagogique, in Environnements Interactifs d'Apprentissage avec Ordinateur, Baron M., Gras R., Nicaud J.F. (eds), Eyrolles, Cachan, 1993, pp. 169180. 
[GRA 92] Grandbastien M., Intelligent Tutoring Systems on Scientific Subjects: Are Prototypes Ready for Broad Experimentation?, Computers and Education, vol 18, ${ }^{\circ} 1$, 1992, pp. 63-70.

[GRA 96] Grandbastien M., Introduction, (numéro consacré à la géométrie), Sciences et Techniques Educatives, vol 3, n², 1996, pp. 145-155.

[GRI 75] Grice H.P., Logic and Conversation, Syntax and Semantics, vol 3, Speech Acts, Academic Press, 1975, pp. 41-58.

[JOS 93] Joshua S., Dupin J-J., Introduction à la didactique des sciences et des mathématiques, Presses Universitaires de France, Paris, 1993, 420 p.

[KRA 82] Kramers-Pals H., Lambrechts J, Wolff P.J., Recurrent difficulties : solving quantitative problems, Journal of Chemical Education, vol 59, $\mathrm{n}^{\circ} 6,1982$, pp. 509-513.

[LEN 95] Lenne D., Le Dialogue en EIAO : Etude à partir de DISCO, un système de conseil en statistique, thèse de l'Université du Maine, 1995, $220 \mathrm{p}$.

[MAS 96] Masseux N., Michau F., Découverte guidée sur simulateur pour l'apprentissage de l'automatique par des élèves-ingénieurs, Sciences et Techniques Educatives, vol 3, nº 1996, pp. 77-100.

[MCC 92] McCalla G.I., The Central Importance of Student Modelling to Intelligent Tutoring, in New Directions for Intelligent Tutoring Systems, Costa E. (ed), SpringerVerlag, NATO ASI Series, 1992, pp. 107-131.

[MEY 94] Mey M., Balas A., Plouin D., Essai sur la maitrise de l'équation-bilan à l'entrée à l'université, Bulletin de l'Union des Physiciens, n766, juil-sept 1994, pp. 1131-1150.

[MOB 92] Möbus C., Pitschke K., Schröder O., Towards the Theory-guided of Help Systems for Programming and Modelling Tasks, in Proceedings of ITS-92, Frasson \& McCalla (eds), Springer-Verlag, 1992, pp. 294-301.

[NGU 93] Nguyen-Xuan A., Joly F., Nicaud J.F., Gélis J.M., Une méthode de diagnostic des connaissances en algèbre pour un module de modélisation de l'élève, in Environnements Interactifs d'Apprentissage avec Ordinateur, Baron M., Gras R., Nicaud J.F. (eds), Eyrolles, Cachan, 1993, pp. 217-228.

[NIC 94] Nicaud J.F. Modélisation en EIAO : les modèles d'APLUSIX, in Didactique et Intelligence Artificielle, Balacheff \& Vivet (eds), La Pensée Sauvage, 1994, pp. 67-112.

[NOE 93] Noël C., Py D., Diagnostic des erreurs des élèves basé sur le contexte, in Environnements Interactifs d'Apprentissage avec Ordinateur, Baron, Gras, Nicaud (eds), Eyrolles, Paris, 1993, 229-240.

[PAC 94] Pachet F., Giroux S., Paquette G., Pluggable Advisors as Epiphyte Systems, in Proceedings of CALISCE'94, Telecom, Paris, 1994, pp. 167-174.

[PAQ 94] Paquette G., Pachet F., Giroux S., Epitalk, un outil générique pour la construction de systèmes conseillers, Sciences et Techniques Educatives, vol 1, n 3, 1994, 305-336.

[PY 95] Py D., Modélisation du diagnosic en EIAO, in Environnements Interactifs d'Apprentissage avec Ordinateur, tome 2, Guin D., Nicaud J.F., Py D. (Eds), Eyrolles, 1995, pp.185-195.

[PY 96] Py D., Aide à la démonstration en géométrie : le projet Mentoniezh, Sciences et Techniques Educatives, vol 3, n², 1996, pp.227-256.

[REI 83] Reif F., How can chemists teach problem solving ?, Journal of Chemical Education, vol 60 , no $11,1983,948-953$.

[SCH 96] Schwob M., Blondel F.M., Questions posées par la conception et la réalisation d'un environnement d'aide à la résolution de problèmes en chimie, Didaskalia, n 8,1996 , pp.115-141.

[SEL 90] Self J., Bypassing the Intractable Problem of Student Modelling, Intelligent Tutoring System : at the Crossroads of Artificial Intelligence and Education, Frasson \& Gauthier (eds), Ablex, NJ, 1990, 107-123. 
[SEL 91] Self J., Formal Approaches to Learner Modelling, Technical report AI-59, Department of Computing, Lancaster University, 1991.

[SEL 94] Selker T., COACH : A Teaching Agent that Learns, Communications of the ACM, vol 37, n², 1994, 92-99.

[STA 90] Stavridou H., Le concept de réaction chimique dans l'enseignement secondaire. Thèse, Université Paris 7, 1990, $240 \mathrm{p}$.

[TAL 92] Talbi M., Joab M., Diagnostic Cognitif de l'Apprenant par Apprentissage Symbolique, in Proceedings of ITS-92, Frasson \& McCalla (eds), Springer-Verlag, 1992, pp. 483-490.

[TIS 90] Tisseau G., Modélisation à partir d'un énoncé informel : le système MODELIS, thèse de l'Université Paris 6, 1990, $430 \mathrm{p}$.

[TRI 96] Trilling L., Rétrospective sur le projet Mentoniezh, Sciences et Techniques Educatives, vol 3, n², 1996, pp.157-162.

[VAN 96] Van Lehn K., Conceptual and Meta Learning During Coached Problem Solving, in ITS'96, Intelligent Tutoring Systems, Third Interntational Conference, Frasson C., Gauthier G., Lesgold A. (Eds), Springer, 1996, pp. 29-47.

[VEI 91] Veillette M., Marcos B., Thérien L., Bourget F., Lapointe J., Modélisation du Diagnostic et des Stratégies d'Intervention dans un Tutoriel Intelligent, Knowledge Modeling and Expertise Transfert, Herin-Aime \& al. (Eds) IOS Press, Amsterdam,1991, 285-298.

[WEN 95] Wentland M., Forte E., Modélisation pédagogique d'un domaine de connaissance : De l'arbre pédagogique d'un texte au réseau conceptuel d'un hyprtexte, in Environnements Interactifs d'Apprentissage avec Ordinateur, tome 2, Guin D., Nicaud J.F., Py D. (Eds), Eyrolles, 1995, pp. 125-136.

[WIN 92] Winkels R., Explorations in Intelligent Tutoring and Help, IOS Press, Amsterdam, 1992, 228p. 